Dorota Chapko

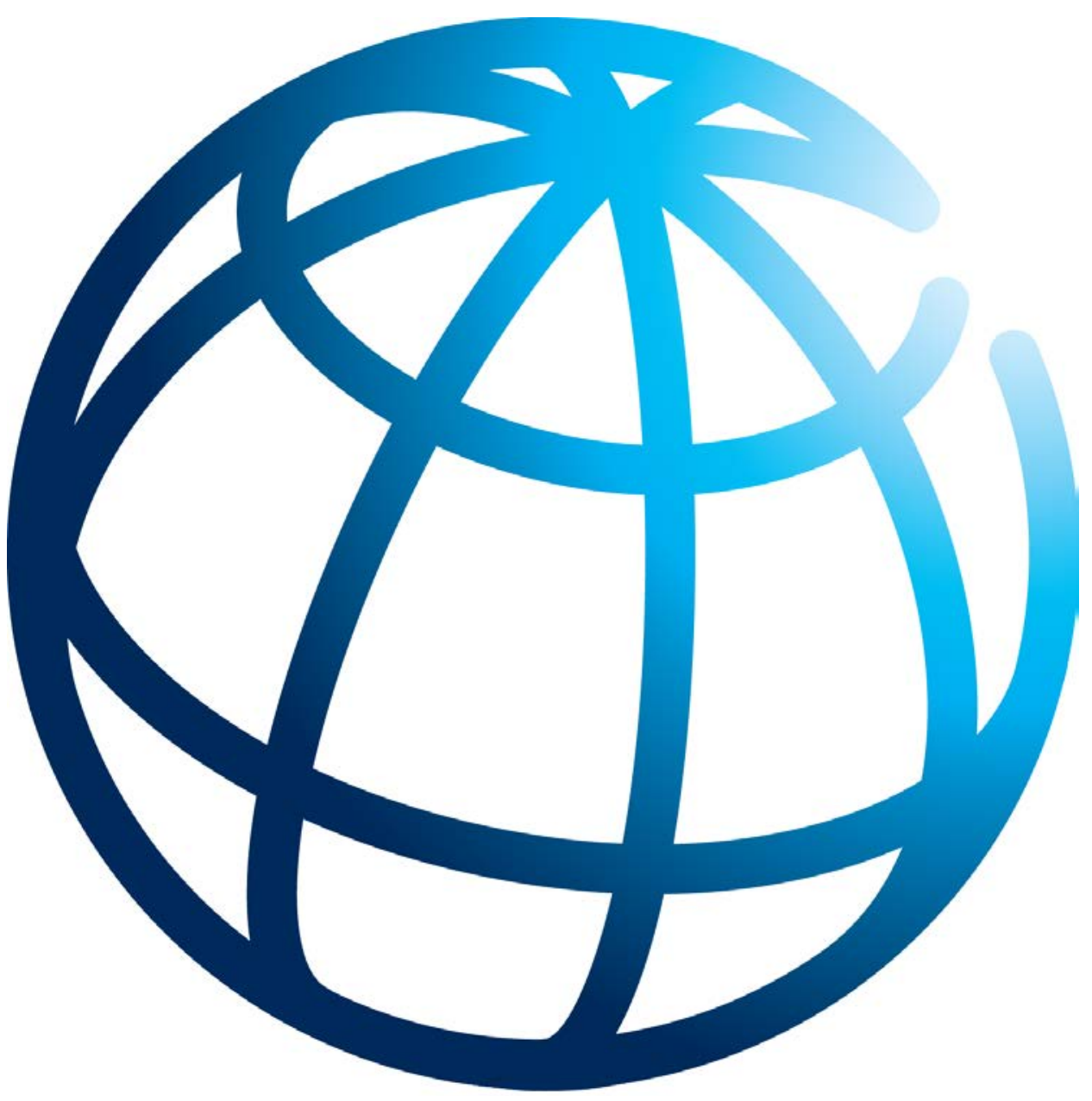





\title{
THE WORLD BANK
}

APPROVED CONCEPT NOTE*

\section{EARLY CHILDHOOD DEVELOPMENT AND SKILLS ACROSS THE LIFE-COURSE THROUGH THE LENS OF THE DEVELOPING BRAIN}

\author{
Dorota Chapko
}




\section{Health, Nutrition and Population (HNP) Discussion Paper}

This series is produced by the Health, Nutrition, and Population Global Practice. The papers in this series aim to provide a vehicle for publishing preliminary results on HNP topics to encourage discussion and debate. The findings, interpretations, and conclusions expressed in this paper are entirely those of the author(s) and should not be attributed in any manner to the World Bank, to its affiliated organizations or to members of its Board of Executive Directors or the countries they represent. Citation and the use of material presented in this series should take into account this provisional character.

The World Bank does not guarantee the accuracy of the data included in this work. The boundaries, colors, denominations, and other information shown on any map in this work do not imply any judgment on the part of The World Bank concerning the legal status of any territory or the endorsement or acceptance of such boundaries.

For information regarding the HNP Discussion Paper Series, please contact the Editor, Martin Lutalo at mlutalo@worldbank.org or Erika Yanick at eyanick@worldbank.org.

\section{RIGHTS AND PERMISSIONS}

The material in this work is subject to copyright. Because The World Bank encourages dissemination of its knowledge, this work may be reproduced, in whole or in part, for noncommercial purposes as long as full attribution to this work is given.

Any queries on rights and licenses, including subsidiary rights, should be addressed to the Office of the Publisher, The World Bank, 1818 H Street NW, Washington, DC 20433, USA; fax: 202522-2422; e-mail: pubrights@worldbank.org.

(C) 2015 The International Bank for Reconstruction and Development / The World Bank 1818 H Street, NW Washington, DC 20433

All rights reserved 


\title{
Early Childhood Development and Skills across the Life-course through the Lens of the Developing Brain
}

\author{
Dorota Chapkoa \\ ${ }^{a}$ Aberdeen Biomedical Imaging Centre, University of Aberdeen, Aberdeen, UK \\ This paper was prepared and funded under the World Bank's Strategic Impact Evaluation Fund \\ (SIEF). SIEF is a multi-donor trust fund created in 2012 with the support of the British \\ government's Department for International Development (DFID) and currently also receives \\ support from the London-based Children's Investment Fund Foundation (CIFF).
}

\begin{abstract}
Human development" and "development" are inseparable, and now new evidence emerges that "brain development" is the key driving mechanism behind this association. The foundations of brain architecture are established early in life. Critical aspects of its structure begin to be shaped by experience before and soon after birth. The current report discusses brain development from a life-course perspective with a particular emphasis on early childhood development (ECD), skills formation, resilience and aging. There is mounting evidence that early and sustained investments in brain development have economic and social returns that can benefit current and future generations. This paper synthesizes knowledge across multiple disciplines and is weighted towards findings from brain sciences to encourage a new perspective on "human development" initiatives among policy makers and international development practitioners. The report discusses the role of social policies in shaping brain function and structure. The policyrelevant findings from brain sciences research can greatly enhance the ability to carry out costeffective policies that foster human development over the life-course, eliminate extreme poverty and improve shared prosperity.
\end{abstract}

Keywords: early childhood development, aging, life-course, brain, skills

Disclaimer: The findings, interpretations and conclusions expressed in the paper are entirely those of the authors, and do not represent the views of the World Bank, its Executive Directors, or the countries they represent.

Correspondence Details: Dorota Chapko, University of Aberdeen, Aberdeen Biomedical Imaging Centre, Lilian Sutton Building, Foresterhill, AB25 2ZD, Aberdeen, UK; tel: +44 (0)1224 438350; email: dorota@abdn.ac.uk or dora12@alum.mit.edu 


\section{Table of Contents}

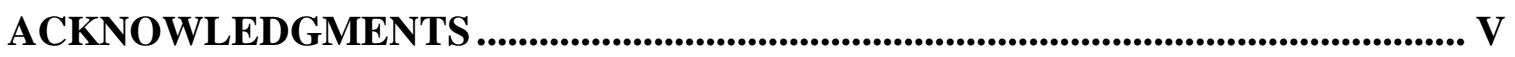

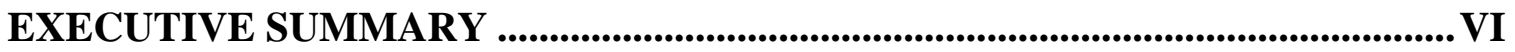

INTRODUCTION: LIFE-COURSE SKILLS DEVELOPMENT WITH THE BRAIN IN MIND ............................................................................................................. VIII

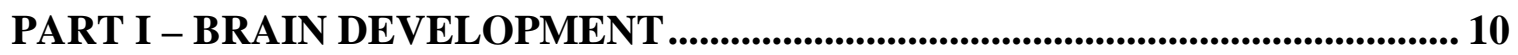

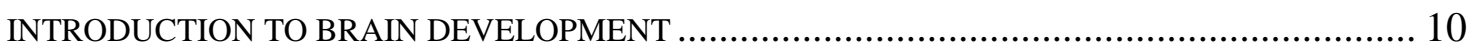

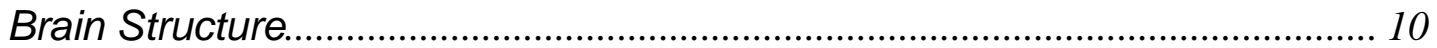

Genetic and Environmental Influences on the Developing Brain .................... 12

BRAIN DEVELOPMENT FROM A LIFE-COURSE PERSPECTIVE .............................................. 13

Prenatal Stage and First Three Years of Life ................................................ 13

From Age Three to Adolescence................................................................ 14

Adulthood and the Aging Brain ......................................................................... 15

BRAIN DEVELOPMENT WITHIN THE CONTEXT OF EXPERIENCE ........................................... 16

Early Plasticity and the Window of Opportunity ................................................. 16

Socio-economic and Psychological Influences on the Developing Brain ...... 17

PART II - SKILLS AND THE BRAIN .......................................................................... 19

NEURAL CONNECTIONS AS THE FOUNDATION FOR SKILLS FORMATION .......................... 19

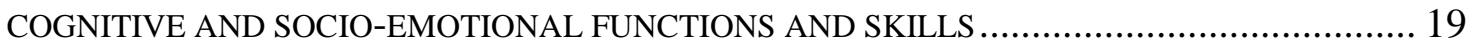

COMPLEMENTARITIES BETWEEN BRAIN FUNCTIONS IN SKILLS FORMATION .................... 20

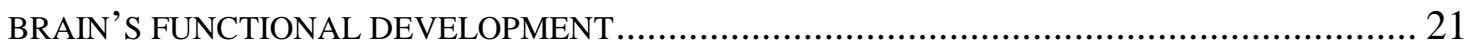

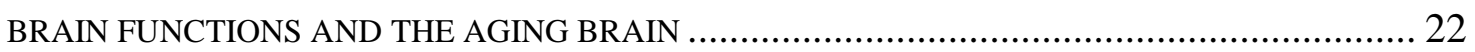

EARLY-LIFE SOCIO-ECONOMIC DISPARITIES IN BRAIN FUNCTIONS............................... 23

PART III - POLICY LEVERS AND BRAIN DEVELOPMENT: ............................... 24

THE ROLE OF SOCIAL POLICIES IN SHAPING BRAIN FUNCTIONS AND STRUCTURE ............. 24 How do Socioeconomic and Psychosocial Influences "get under the Skull"?

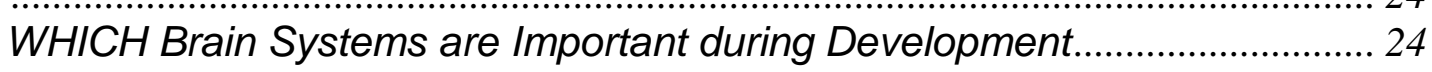

WHEN are Disparities in Neural and Cognitive Development first Detected 25

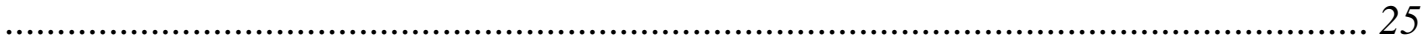

WHO Should Constitute the Priority Target Group........................................ 26

BRAINS RESILIENCE AT THE CORE OF SOCIAL POLICIES ................................................ 29

Recovery in Brain Structure and Function ...................................................... 29

Harnessing the Power of the Human Brain ........................................................ 30

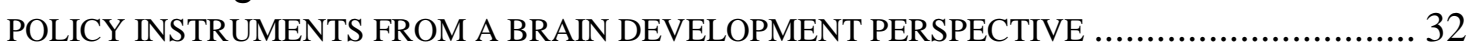

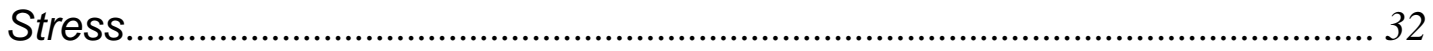

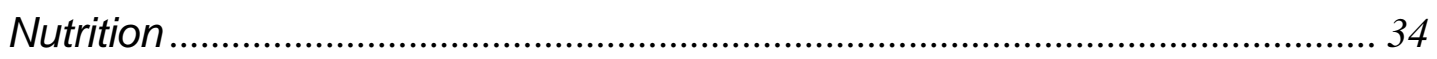

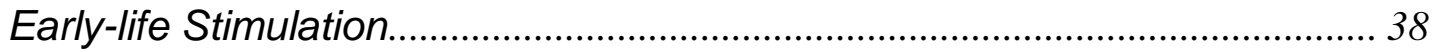

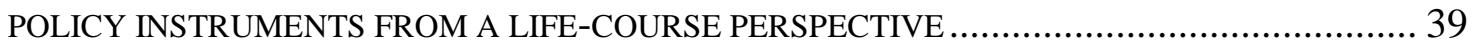

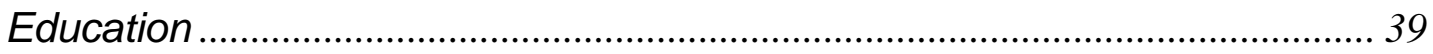

Job-relevant Skills...................................................................................... 40 
CONCLUSIONS AND POLICY IMPLICATIONS............................................. 42

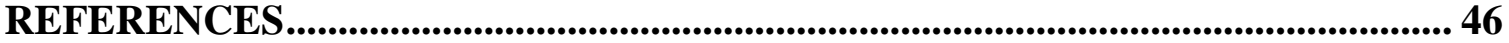




\section{ACKNOWLEDGMENTS}

This work was prepared and funded under the World Bank's Strategic Impact Evaluation Fund (SIEF). The paper was authored by Dorota Chapko. Julieta Trias, Economist, (World Bank), was the Task Team Lead for this project. We are grateful to our peer reviewers and colleagues for their valuable comments and contributions. The peer reviewers included Emanuela Galasso, Senior Economist, Sophie Naudeau, Senior Education Specialist, Omar Arias, Lead Economist, (World Bank), and Professor Alison Murray, (University of Aberdeen, UK).

We are especially thankful for comments received from our SIEF colleagues including Joost de Laat, a former Program Manager, and Aliza Marcus, Senior Communications Officer, and other colleagues across the World Bank including Claudia Costin, Senior Director, Bénédicte Leroy de la Brière, Lead Economist, and Lucy Bassett, Social Protection Specialist. In addition, we received valuable insights through a workshop organized as a background input to this paper. We would also like to thank Holly Blagrave, Consultant, and Rachel Rosenfeld, Consultant, (World Bank), for administrative support of the project. 


\section{EXECUTIVE SUMMARY}

It's widely recognized that achieving the brain's full developmental potential, starting from early childhood, is crucial for socio-economic success. Brain complexity -i.e. the diversity and complexity of neural pathways and networks- is moulded in a person's early-life and has an impact on the development of cognitive and socio-emotional human abilities. Early life experiences affect childhood and later life-course development through changes in brain structure and function. The first years of life starting in-utero is a window of opportunity for investments that will lead to a healthy and productive life. Jim Heckman, Nobel Laureate in economics, and his collaborators have compellingly shown that strong foundational skills built in early childhood lead to a "self-reinforcing motivation to learn" so that "skills beget skills" (Heckman 2006). This ultimately leads to betterpaying jobs, healthier life-style choices, greater social participation, and more productive societies.

This work calls for an approach to human development policies that integrate the domains of early childhood development (ECD), skills formation, aging, and resilience within a lifecourse framework. First, "human development" and "development" are inseparable, and now new evidence emerges that "brain development" is the key driving mechanism behind this association. Second, development and aging are part of a continuum; the positive and negative early-life experiences may have profound consequences in later-life due to their accumulating impact over the life-course. This paper synthesizes knowledge across multiple disciplines and is weighted towards evidence from brain sciences to encourage a new perspective on "human development" among policy makers and international development practitioners.

Brain development across the life-course goes beyond the behavioral science toolkit and calls for neuroscience-informed interventions that would complement behaviorallyinformed initiatives. This report encourages thinking about behavioral change from a developmental and life-course perspective by recognizing that decision-making processes across the life-course are rooted within the developing brain - therefore complements the discourse on neuroeconomics and decision making processes (Camerer, Loewenstein \& Prelec 2005).

\section{Key messages:}

- Positive and negative experiences in early-life affect childhood development through changes in brain structure and function. A healthy and active brain, shaped by adequate nutrition and safe and enriching environments starting in utero, enables retention of brain functioning across the lifespan and healthy brain aging.

- Both cognitive and socio-emotional abilities are vital to successful early childhood development. Evidence from brain sciences shows that "cognitive" and "non-cognitive" or rather "socio-emotional" functions are interdependent (Pessoa 2008). Both cognition and emotions are governed by a number of overlapping neural systems which complement each other. Together they give rise to higher order brain processes which underline complex skills; it is thus important to ensure that interventions targeting human development and skills acquisition are holistic and target both cognitive and socio-emotional domains.

- Multiple studies from low-, middle- and high-income countries have shown that early childhood socio-economic circumstances are associated with brain functions across the life-span, staring in early childhood. To eliminate the socioeconomic disparities in brain function, we need to understand the neural pathways of how early life-conditions or its facets, that is, specific modifiable factors (for example, nutrition, early stimulation) "get under the skull". With this knowledge, we will be better equipped to design and evaluate effective and scalable initiatives aimed at reducing life-course individual differences in brain functioning (Noble, Farah 2013). 
- WHICH brain systems are important during development? Recent studies have indicated that brain systems affected by early-life socioeconomic adversities play a critical role in functional domains essential for later school achievements (Brito, Noble 2014) such as language skills, working memory, cognitive control, mental flexibility, self-regulation skills and an overall ability to form meaningful relationships.

- WHEN disparities in neural and cognitive development can be detected? The resulting socioeconomic differences in neural development and brain functioning begin to emerge already in infancy; if no intervention is provided at the right point in time, they persist and even widen with increasing age. Generally, because of the brain's decreasing malleability across the life-course, the earlier sustained and high quality experiences impact the brain, the better for the brain.

- WHO should constitute the priority target group? Everyone deserves a good head-start and the attainment of optimal brain development. Individuals from the most disadvantaged backgrounds, who will have greatest developmental delays and brain burden, need the earlylife investment the most.

- Brain complexity programmed in early life facilitates retention of brain functioning across the lifespan and healthy brain aging. Optimal brain development provides an individual with a greater number of neurons (the biological units of the brain), more synapses (connections between neurons), and thus multiple pathways to perform any given task. Such "neuronal redundancy" comes in handy when faced with deleterious brain aging. An increasing number of studies suggest that early childhood interventions targeting mental domains might increase maximum life-time cognition, potentially reduce the trajectory of cognitive decline in late-life, and even postpone the point at which cognitive deficits first appear.

- The human development policies should be implemented within the life-course framework. Many of the qualities of brain resilience to biological and social factors are shaped in early life when the brain is most plastic. With the appropriate investments extending beyond early-life, for example, in quality education and jobs, resilience can be sustained across the life-course. Therefore maximum brain development should be sustained with life-course investments and equal opportunities for highest returns.

- The associations between SES and brain function and structure could emerge from disparities in the prenatal environment as well as postnatal experience or exposures. Potentially modifiable and policy-relevant life-course factors include stress reduction, nutrition, early-life stimulation, education and job-relevant skills.

- Multi-factor interventions are the key. Because children in developing countries are exposed to multiple risks to brain development at once, it is very unlikely that a single-factor intervention will solve the issue of suboptimal brain development with its life-course consequences; rather, multi-factor interventions incorporating the features of behavioral change might produce the expected results (Aboud, Yousafzai 2015).

In summary, mounting evidence from studies across several disciplines - neuroscience, health, education, economics, and psychology - provide evidence that early and sustained investments in human development are the key for our neurons, our brains, for us as individuals, and for our societies. They lay the foundations for our capacity to achieve and to function well despite social or even biological obstacles and adversities throughout the life course. 


\section{INTRODUCTION: LIFE-COURSE SKILLS DEVELOPMENT WITH THE BRAIN IN MIND}

It's widely recognized that achieving the brain's full developmental potential, starting from early childhood, is crucial to socio-economic success and well-being. Brain complexity programmed in a person's early-life (that is, the diversity and complexity of neural pathways and networks), gives rise to cognitive and socio-emotional functions which fuse together to form human abilities acquired across the life-course (Richards, Hatch 2011). Advances in the brain sciences show that, in addition, individuals with a good head-start in brain development are more resilient to potential mid-life adversities and the aging process. This is important because globally by 2050 there will be 400 million people aged $>80$ years. Societies around the world in both developed and developing countries face extensive complex challenges when developing the needs of the increasing number of elderly. These demographic changes call for changing focus of the interventions from "survival" to "thriving" (World Bank 2015) and an approach to human development policies that integrates the domains of early childhood development (ECD), skills formation, aging, and resilience within a life-course framework (Richards, Hatch 2011). This paper synthesizes knowledge across multiple disciplines and is weighted towards evidence from brain sciences to encourage a new perspective on "human development" initiatives among policy makers and international development practitioners.

Early brain development is like building a house - just like a house needs a strong foundation, early stages of brain development provide a foundation for more complex brain architecture, behaviors, and successful early childhood development (ECD) which covers the period from pregnancy until a child transitions to primary school (and up to age 8). During early childhood, the brain undergoes enormous growth and rapid development and is most malleable to experiences, especially in the first 2-3 years of life. Thus the first years of life starting in-utero is a window of opportunity for investments that will result into a healthy and productive life. A healthy and active brain, shaped by adequate nutrition and safe and enriching environments in early-life (Walker et al. 2007), enables retention of brain functioning across the lifespan and healthy brain aging. Jim Heckman, Nobel Laureate for economics, has compellingly shown that strong foundational skills built in early childhood lead to a "self-reinforcing motivation to learn" so that "skills beget skills" (Heckman 2006). These can ultimately lead to better-paying jobs, healthier life-style choices, greater social participation, and more productive societies. Therefore the achievement of an optimum neurodevelopment and therefore maximum brain skills across the life-span should be a priority policy area in all countries around the world.

Early childhood development is complex and relies on multiple systems and organs; however, it is widely recognized that early-life experiences affect childhood development mainly through changes in brain structure and function. The availability of positive experiences help the brain grow and thrive across the life-course. The presence of negative experiences hurt brain development and impede a child's ability to flourish into a successful adult; "Poverty's most insidious damage is to a child's brain" (Luby 2015). This is especially alarming today when poverty rates are highest among children with some 400 million still living in extreme poverty (World Bank 2013).

Multiple studies from low-, middle- and high-income countries have shown that early childhood conditions as estimated by socio-economic status (SES), poverty, or more specific elements of SES are correlated with brain functions across the life-span, starting in early childhood. Few of them are able to estimate the causal effect. To eliminate the socioeconomic disparities in brain function, we need to understand the neural pathways of how early life-conditions (SES, poverty) or its facets, that is, specific modifiable factors such as nutrition, stimulation, attachment "get under the skull." With this knowledge, we will be better equipped to design and evaluate effective and scalable initiatives aimed at reducing life-course individual differences in brain functioning. Although this field is still in its infancy, recent studies have indicated that brain systems affected by early-life socioeconomic adversities play a critical role in functional domains essential for later school 
achievements. Unsurprisingly then, our main goal as global citizens should be to foster and protect children's developing brains (Luby 2015).

The current report presents the themes of early childhood development (ECD), skills formation, aging and resilience within an integrated life-course framework through the lens of the developing brain. By investing in early childhood neurodevelopment, "cognitive", "socio-emotional" as well as social and economic returns might be present immediately and decades later, this way contributing to greater well-being of individuals in development, maturation and aging. The first part of the report discusses how socio-economic success and healthy aging begin early in life with the developing brain. Next, the concept of resilient brain aging in neuroscience is introduced and the catalytic role of an adequate early-life environment and a brain developed to its full potential in building cognitive and socio-emotional abilities and a more resilient brain. Finally, potential policy levers with their implications on the brain across the life-course are described. While details of how to intervene are beyond the scope of this report, some examples of already implemented interventions presented from a life-course perspective are included. Overall, the policy-relevant findings from brain sciences research can greatly enhance our ability to carry out cost-effective policies that foster human development over the life-course, eliminate extreme poverty and improve shared prosperity. 


\section{PART I - BRAIN DEVELOPMENT}

\section{INTRODUCTION TO BRAIN DEVELOPMENT}

\section{Brain Structure}

To understand how the developing brain influences brain structure and functions in later-life, we have to understand how the brain's morphology changes as well as how the output in the form of cognitive and socio-emotional functions changes across the life-course. In order to understand the links between skills development and the brain in greater detail, it is important to provide first a short introduction to the brain itself (Gazzaniga, Ivry \& Mangun 2009).

Neurons are at the center of learning, memory and communication. A neuron is a biological cell which constitutes a basic signaling unit within the brain. It consists of structures called a cell body, dendrites and an axon. Dendrites, which are treelike processes extending from the cell body of a neuron, receive information from other neighboring neurons. Based on this information neurons make a "decision" on what to do next and this communication results in a network of millions of neurons. Specifically, neurons communicate by firing an electrical nerve impulse along the axon; by changing their activity levels, neurons send a signal to their neighbors at locations called synapses - a small gap between the axon terminal of the sending neuron and the dendrite of the receiving neuron. When electrical signal arrives at a nerve terminal, chemicals called neurotransmitters are released from the axon, then bind to the receiving molecules on the dendrites of the receiving neuron, and transmit impulses from one neuron to the next. Myelin, a fatty substance wrapped around the axons of many neurons, enhances the speed of the information flow between neurons by helping to transmit electrical impulse along the axon. Myelination is the process of wrapping the axons of neurons with myelin.

Neurons make functional connections with each other to establish neural networks which support all brain processes, including cognitive and socio-emotional functions. The outer part of the brain mainly consists of the neuronal cell bodies (grey matter) and the inner layer of the brain mainly consists of nerve fibers connecting different brain areas (white matter). Glial cells are neurons' supporting cells which facilitate the speed of information transfer and generally provide structural support. In fact, glial cells greatly outnumber neurons by a factor of 10 and may constitute more than a half brain volume.

The human cerebral cortex is at the center of cognitive processes and is viewed as "the biological substrate of human cognitive abilities" (Rakic P, Arellano JI, Breunig JJ 2009). The human cerebral cortex is the brain's outer layer which mainly consists of the grey matter. It occupies two symmetrical hemispheres and it is a highly organized layered structure. The cerebral cortex is $\sim 2$ -4 millimeters thick and has a highly folded surface tissue: if you were to spread it you would create the sheet of $\sim 1 \mathrm{~m}^{2}$. Cortical surface area and cortical thickness have distinct features and different developmental trajectories. Interestingly, from an evolutionary perspective it seems like the addition of new functions to the cortex requires significant expansion of cortical surface and relatively small enlargement of cortical thickness; in fact, human's cortical surface area is a thousand fold greater than that of the mouse whereas cortical thickness is only 2-3 times larger (Rakic P, Arellano Jl, Breunig JJ 2009). The role of the cerebral cortex is to receive external information from the "subcortical" structures located below the cortex, subsequently to integrate this information within and across different cortical functional areas and finally send the processed information to other brain regions to initiate the most appropriate behaviors. The cerebral cortex is organized as a map and is divided into 4 lobes which reflect the anatomical organization of functional areas: the frontal, parietal, temporal, occipital. Occasionally, researchers identify the $5^{\text {th }}$ lobe - the limbic lobe. Apart 
from the cerebral cortex visible on the surface of the brain, other important subcortical structures are involved in cognitive and socio-emotional processing. For details, see Box 1.

Box 1: Brief Description of the 4 Basic Lobes (Gazzaniga, Ivry \& Mangun 2009):

- The frontal lobe controls planning, executive functions (cognitive control), and execution of movements. It is further divided into the motor cortex and the prefrontal cortex (PFC). PFC is very essential for the more complex aspects of planning and executing behavior which require the integration of information over time. It is also central to emotional regulations.

- The parietal lobe receives sensory information about touch, pain, temperature, limb position and controls coding space and coordinating actions.

- The temporal lobe is engaged in auditory, visual, multimodal processing and language comprehension.

- The occipital lobe is involved in processing of visual information.

Apart from the cerebral cortex visible on the surface of the brain, other important subcortical structures are involved in cognitive and socio-emotional processing. Below we highlight only those brain components which have been implicated and highlighted in the literature on early childhood development or brain maturation and aging. Keep in mind that the brain is far more complex than that and consists of many more subdomains!

- Hippocampus is located in the temporal lobe of the brain and its shape resembles a seahorse. Humans have two hippocampi. Hippocampus is central to learning and memory. As we learn, the hippocampi support the rapid consolidation and initial storage of information for some memory types. It is also important for the formation of new long-term memories. It is abundant with glucocorticoid receptors implicated in stress responses.

- Amygdala composes of groups of neurons composing an almond-shaped structure within the temporal lobe. It is an integral part of the "limbic system" and mainly implicated in emotional functioning and learning such as responses to negative social events or threatening emotional situations.

- Hypothalamus is a collection of nuclei important for the maintenance of homeostasis i.e. maintaining the stability of the body. The hormones produced by the hypothalamus control the endocrine system and the autonomic nervous system which initiates a "fight or flight" behavioral response. It receives inputs from the limbic cortex and is involved in emotional processing.

Occasionally, researchers identify the $5^{\text {th }}$ lobe - the limbic lobe. The limbic system is involved in emotional processing, learning, and memory. The main limbic brain areas including PFC, amygdala, and hippocampus regulate neuroendocrine, autonomic, and immune systems and therefore are central to stress responses (McEwen \& Gianaros, 2010). While PFC and hippocampus reduces stress responses, amygdala intensifies this process. Stress initiates the release of a hormone produced by the adrenal glands called "cortisol" which binds to the glucocorticoid receptors. Excess cortisol levels are toxic to neurons which are very sensitive to biological insults underpinning stress; especially to hippocampus which is abundant with glucocorticoid receptors. If the release of cortisol is prolonged and becomes chronic, then the brain's regulatory responses to stress might be maladaptive.

Corpus callosum is a neural fiber area which connects the left and right cerebral hemispheres facilitating communication between the two regions. 
Achieving the brain's full developmental potential is crucial for a lifetime of success. Early life experiences impact childhood development through changes in brain structure and function (Walker et al. 2007). Early brain development is like building a house - just like a house needs a strong foundation, early stages of brain development provide a foundation for more complex brain architecture, behaviors, and successful early childhood development. It is cumulative - even a minor error will have an impact on the subsequent stages of development (Nelson et al. 2014) including maturation and aging. During the first years of life brain undergoes enormous growth and rapid development. The brain is most malleable in early childhood i.e. the brain's ability to change in response to experience is greatest. However, brains are built over time from few weeks after conception through adolescence and early adulthood (Nelson et al. 2014). Therefore the brain needs supporting and nurturing environments that give rise to positive experiences for optimal development so that the child's brain can reach its full potential for lifelong success.

What does it mean to reach the brain's full developmental potential? All components of mental health including morphology and function of the brain are in part genetically determined and regulated by the unchangeable DNA sequence. For example, brain size is almost entirely genetically determined (Bartley, Jones \& Weinberger 1997). Therefore genes act as "the initial blueprint" for the developing brain and "the template for brain architecture" (Nelson et al. 2014). However, brain structure is predominately environmentally determined (Bartley, Jones \& Weinberger 1997). The environment and associated experiences are important for "fine-tuning" of the brain and therefore play an important role in early childhood development (Nelson et al. 2014). Because optimal brain development requires the presence of a high quality experience, regardless of the genetic background, a child's brain will not reach its full developmental potential if deprived of high quality environmental inputs.

The brain needs experience for optimal development: "if starved of experience, then the journey of development has little structure" (Nelson et al. 2014). Experience is defined as the interaction of the brain with the environment. Although genetics play an essential part during development, experience-driven modifications within the brain shape neural connections within constraints imposed by genetic background (Nelson et al. 2014). The prolonged phase of development in humans, in comparison to other mammals, and the brain's extraordinary power of malleability during development means that the human brain has a greater window of opportunity to adjust to specific environments which are the source of specific experiences. Therefore the brain achieves its full potential within constraints imposed by the genetic background and the availability of experiences.

The environment and the associated experiences can influence how genes are expressed via epigenetic alterations. For example, DNA methylation is a chemical change in DNA which determines how genes are expressed by turning a gene on or off. Certain environmental conditions can change methylation of specific genes, even in utero (i.e. in the womb), with effects present across the life-course (Richards, Hatch 2011). DNA methylation is dependent on folate, vitamin B12, and vitamin B6 therefore nutrition can cause epigenetic changes; in fully differentiated neurons epigenetic modifications support neuronal functions and malleability. Insults such as infections, maternal alcohol, nutrition restriction, and endocrine changes due to maternal stress might result in dysregulation to fetal neurodevelopment and an atypical architecture of the brain (Van den Bergh 2011). 


\title{
BRAIN DEVELOPMENT FROM A LIFE-COURSE PERSPECTIVE
}

\author{
Prenatal Stage and First Three Years of Life
}

The prenatal stage, the most rapid phase of brain development, provides the structural framework for subsequent development and modification of neural networks. The brain starts developing soon after conception and the biological mechanisms governing these processes frequently intersect in time and space. The core of brain architecture is formed between day 56 post-conception until about 24th week of gestation (Van den Bergh 2011). Neurons with their supporting glial cells are generated in the first half of gestation (Rakic P, Arellano JI, Breunig JJ 2009). Classical studies established that almost all cortical neurons in human are generated in utero. Interestingly, this suggests that the same neurons operate highest cognitive and socioemotional functions across the entire life-span highlighting the importance of optimal brain development before birth (Rakic P, Arellano JI, Breunig JJ 2009). At the start, neurons and glial cells massively migrate to their destined layers of the cerebral cortex starting with the deepest layer first and moving on to the most outer ones. Then, from 8 to 16 weeks of gestation, accumulating cells in the cerebral wall form "the primitive brain" (Nelson 1999) - a construct that will give rise to the mature cerebral cortex; at first transient and then increasingly more permanent neuronal layers and connections between subcortical structures and the cortex are made. Subsequently, between 24 and 28 weeks of gestation, all the neural connections are refined and this process continues until perinatal period which extends beyond birth. The transiently overproduced neurons, axons and synapses die while the remaining axons and dendrites grow to make new topographical connections in the cortex what will serve as the base for functionally coordinated and complex outputs in the years to come.

From about 34 weeks of gestation to about 2-3 years after birth, the brain undergoes enormous growth and development. While it has been established that most cortical neurons in human are generated in utero, the formation of new neurons continues well after birth in other brain structures such as hippocampus and cerebellum (Rakic P, Arellano Jl, Breunig JJ 2009). It is when the brain is most plastic, that is, most able to make new connections between neurons (see Figure 2). During this period new neural connections are formed at a tremendous rate - in fact, synapse addition approximates a logarithmic growth with 40,000 synapses being generated every single second to reach the level of 1,000 trillion connections by age three! Overall, these processes reach their peak by two years of age and play an important role particularly in speech and verbal communication formation, highlighting the importance of the first 1,000 days of life (Levitt 2003); (Rakic P, Arellano J.I, Breunig J.J 2009)). Because the brain's malleability decreases exponentially throughout the years, this period provides the opportunity to change the brain's architecture with minimal effort (see Figure 2). In practical terms, during early years the brain can easily "absorb" positive experiences to develop properly or is especially vulnerable to environmental insults which impinge on the developing brain - the developing brain needs to be simultaneously nurtured and protected for optimal outcomes to occur. 
Figure 2: Malleability of the Brain Across the Life-course

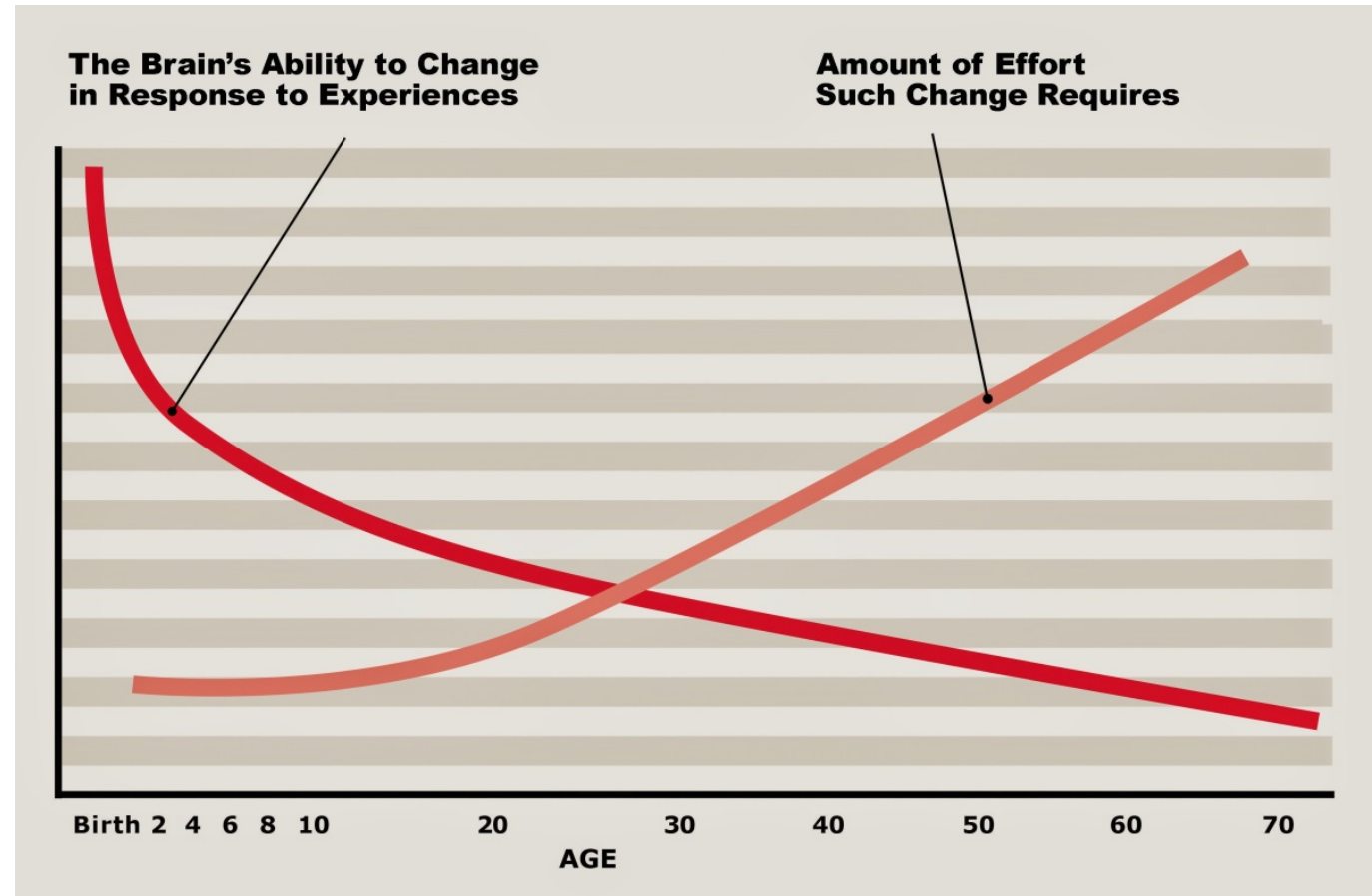

Source: Pat Levitt (2009) in http://developingchild.harvard.edu/science/key-concepts/brain-architecture/

\section{From Age Three to Adolescence}

Brain development is cumulative and continues through childhood and adolescence. Even though between ages 3-6 the new connections between neurons are formed at a slower rate than in the first three years of life, it is in later childhood and adolescence when brain areas responsible for most complex brain functions progressively increase their involvement in development. The loss of unnecessary connections continues until adolescence. In fact, during puberty, it is estimated that synapses in the human cerebral cortex are lost at the rate of 100,000 per second! (Nelson, 2010). This ensures that the brain spends energy on the fine-tuning and the improvements of the necessary pathways (Nelson, 2012 and Nelson, 2010).These changes are as remarkable and important as the ones that occur before age 3 (Nelson et al. 2014) and allow for further maturation and sophistication of neural processes (Rakic P, Arellano J.I, Breunig J.J 2009).

The developing cortex undergoes substantial changes through adolescence. The cortex increases in size through adolescence mainly due to the net increase in number of synaptic connections and due to glial development and myelination of nerve fibers which constitute the final stages of brain development (Kolb, Gibb 2011). The process of myelination requires our particular attention because normal adult brain functions are attained only after myelination is complete; in essential brain regions, such as PFC, this is achieved only after 18 years of age (Kolb, Gibb 2011). In contrast to white matter, gray matter increases from birth to age 4 to 5 years and then gradually decreases in a non-uniform fashion across the cerebral hemisphere. The subdomains of grey matter, cortical surface area and cortical thickness, have also distinct developmental trajectories in normal development. On the one hand, the cortical surface expands until adolescence and then decreases in adulthood. On the other hand, the cortical thickness declines rapidly in childhood and undergoes the process of gradual thinning to reach its plateau in early adulthood (Noble et al. 2015). 
With age our brains accumulate pathologies (sub-clinical disease) which erode structural and functional brain integrity. The deterioration of grey matter and white matter as well as subcortical structures underpins aging processes and dementia (For details, see Box 2). Even though there are conflicting results regarding the extent to which loss of neurons (atrophy) occurs in old age (Whalley et al. 2004), reduction in brain size and the acceleration of brain atrophy, especially in the prefrontal regions (Deary et al. 2009) and hippocampus (Murray et al. 2011), have been generally established. Furthermore, the density of neural connections in the frontal cortex drops and this decrease is greater in patients with Alzheimer's disease (Small, Mok \& Bornstein 2001) (For details, see Box 2). In general, shrinkage of the cerebral cortex is less pronounced for the nerve fibers connecting different brain areas (white matter) than for the neuronal cell bodies (grey matter) and cortical thickness (Deary et al. 2009). Brain white matter volume tends to be relatively preserved until about age 70 when it follows a steep trajectory of decline (Deary et al. 2009). This may result in worsening of connectivity between cerebral areas (Whalley et al. 2004). Another important aspect of structural brain changes in aging is the presentation of white matter hyperintensities (WMH); these "lesions" are depicted as areas of high signal intensity at magnetic resonance (MR) imaging. WMH are vascular in nature brain abnormalities acquired across the lifecourse and may reflect age-related disturbances in brain metabolism that contribute to the lifetime burden of brain aging (Murray et al. 2005); it is the determinant of global functional decline in older adults.

\section{Box 2: "The Aging Brain and Dementia"}

Cognitive abilities might decline to the point at which detectable deficits occur potentially leading to the diagnosis of cognitive disorders such as dementia. Dementia is a clinical syndrome that can be due to various pathologies that affect the brain. It is the leading cause of loss of independence and requirement for institutional care and dementia incidence increases exponentially with age. However, it is not an inevitable consequence of aging and is superimposed on normal age-related slowing of cognitive processing speed. Alzheimer's Diseases (AD) is the most common cause of dementia. $A D$ is characterized by the build-up of two damaging proteins in the brain called $\beta$ amyloid $(A \beta)$ and tau; while $A \beta$ accumulates around neurons, tau collapses into neurofibrally tangles inside a dying cell. Cerebrovascular disease is another most common cause of age-associated structural changes and neuropathologies (Matthews et al. 2009). It includes a spectrum of conditions underlined by problems with the blood supply to the brain such as stroke or transient ischaemic attacks (TIAs). Both AD and cerebrovascular disease can cause cognitive impairment and dementia.

With the increasing life expectancies around the globe, the aging processes are prolonged and there is more chance for individuals to cross the threshold for clinical expression of dementia. The number of people living with dementia worldwide is currently estimated at 47 million and this number is predicted to triple by 2050 (Prince, M, Wimo, A, Guerchet, M, Ali, G, Wu, YT, Prina, M, 2015). Dementia prevalence differs by socio-economic status and occurs more frequent in those from disadvantage backgrounds. Importantly, nearly 60 percent of dementia cases live in low- and middle-income countries. There is currently no treatment for dementia and dementia related care is costly and complex; some of the main challenges associated with dementia include the economic impact of dementia on families, caregivers and communities, and the associated stigma and social exclusion (World Health Organization, 2012). In March 2015 WHO hosted the first Ministerial Conference on Global Action Against Dementia to raise awareness of the global socio-economic burden created by dementia and to move the global dementia agenda forward. 


\title{
BRAIN DEVELOPMENT WITHIN THE CONTEXT OF EXPERIENCE
}

\author{
Early Plasticity and the Window of Opportunity
}

Neuroplasticity is the mechanism by which positive and negative experiences change and reorganize neural connections. The extended period of brain development involves neuronal and synaptic overproduction and their subsequent elimination. This is because neurons and synapses await confirmation from the environment in the form of an experience. In the cerebral cortex, neurons and connections between them are overproduced during development in the processes called "neurogenesis" and "synaptogenesis" respectively. The peak of maturation is marked by cell death and a substantial reduction of synapses in the process called "neuronal apoptosis" and "synaptic pruning" respectively. Just like in the process of creating a statue with a block of stone the unwanted pieces are removed to achieve the desired function and shape, the brain has a parallel system in which unneeded neurons and synapses are removed (Kolb, Gibb 2011). The purpose of these mechanisms is to capture and incorporate experience into the developing architecture of the brain.

Synapse formation and synaptic loss constitute the basis for neuroplasticity in development and beyond (Rakic P, Arellano J.I, Breunig J.J 2009). Synaptogenesis and pruning are guided by environmental cues and contribute to individual differences in neural circuits of the cerebral cortex (Kolb, Gibb 2011). This synaptic change is the form of experience-induced neuroplasticity underlying skills development. Most areas of cerebral cortex massively overproduce synapses in the 1st year of life. Synapses which are overproduced during early development "expect" specific experiences which in turn can prune them back to the optimal adult levels throughout the cerebral cortex - therefore in early-life these synapses are "experience-expectant" (Kolb, Gibb 2011) (see Box 3). Experience, positive or negative, activates certain neural pathways; with repeated experience, the same neural pathways underlying certain skills are used multiple times (Noble 2014). In this process the neural connections along these activated pathways are confirmed - these connections are strengthened and stabilized. Connections that are not involved under this experience are not confirmed - these connections are eliminated. Synaptic plasticity in early childhood requires high quality experiences which are repeated over time for an optimal outcome.

Early stages of development are characterized by "windows of opportunity" during which different areas of the brain are sensitive to experience at different time points. First, in the neurodevelopmental neurosciences, a "critical period" refers to a narrow time frame during which a specific experience must take place for an expected developmental outcome to occur. Second, a "sensitive period" encompasses a somewhat extended time period from the one imposed by the "critical period" and therefore is more flexible in nature. In fact there are only few "critical periods" in human development (Thompson, Nelson 2001). For example, an infant must be exposed to linguistic input in the form of speech or sign language during the first year of life for normal language acquisition (Petitto 2005) (Thompson, Nelson 2001). However, time intervals within early childhood development are better represented as "sensitive periods" (Nelson 1999). For example, the timeframe for forming meaningful attachments to caregivers is more open-ended; even in case of a child experiencing profound neglect in early-life there is still high possibility that he or she will develop an attachment relationship with a potential future caregiver (Nelson 1999). The distinction between "sensitive" and "critical" periods is important because "sensitive periods" imply some hope for "catch-up growth" in different developmental domains and the possibility for effective early childhood development interventions.

The availability of a specific experience during a "sensitive period" which activates the "experience-expectant" synapses is the key feature of early brain development. However, the elements of the expectable environment must be present at the right time (Nelson et al. 2014). If the right experience is not available at the right time, that might lead to over-pruning or no pruning at all, which is equally damaging. As the result, the developing brain could end up with too few neurons and synapses. It is not only the presence of an experience at the right time that is 
important. Only a high quality experience may lead to desirable developmental outcomes. A faulty input may result in maladaptive brain development. For example, if a caregiver feeds a child (the availability of experience) while both face away from each other (a faulty input), then a warm and trusting relationship may not fully developed (maladaptive brain development) (Nelson et al. 2014). Overall, the brain might not reach its full developmental potential from lack of experience or faulty, low-quality input.

Box 3: "experience expectant" vs "experience dependent" information storage during early childhood development and beyond (Greenough, Black \& Wallace 1987)

In general, brain development is characterized by experience-expectant information storage and later brain development and adulthood relies on experience-dependent information storage. The "experience-expectant" storage of information is common across mammals. Throughout the evolutionary history, mammals relied on predictable experiences which they have expected during development - the expectation of these experiences has stayed with humans until today. Within the experience-expectant paradigm, the expected experiences prune synaptic connections back to the adult level - this way the performance of brain systems can be appropriately tuned to the given environment for an optimal performance. The loss of synaptic connections can actually make a less organized pattern into a highly organized one. This type of information storage is characterized by a restricted period and therefore constitutes the essence of the sensitive period. Due to individual differences (e.g. underlined by genetic predispositions), in the presence of an identical experience, brain structure can be altered differently. It is not only the quality of experience that determines the changes in brain structure and function, but also the intrinsic characteristics of an individual. In addition, an infant is not a passive recipient of the expected experience but also has a role to play in controlling the experiences produced by a caregiver. Later in life, the synaptic changes are induced to specific experiences and are more local therefore those synapses are not "experience-expectant" but more "experiencedependent". Even though synapses overproduction and pruning seem to dominate early years, experience-dependent synaptic growth accompanies this process to some extent as well.

Socio-economic and Psychological Influences on the Developing Brain

Early childhood development does not occur within a vacuum; this complex process is influenced by the interplay between individual characteristics, biological forces, family and class dynamics imbedded within broader historical, socio-cultural and economic circumstances and other environmental factors (Boyden, Dercon \& Singh 2014). These conditions in turn determine the availability, quantity and quality of a wide range of positive and negative experiences which impact early brain development, as broadly discussed in the previous section. The idea that not only biological but also socio-cultural and economic experiences (determinants) influence health outcomes has been a topic of extensive and passionate research across multiple disciplines, including sociology, anthropology, psychology, epidemiology, animal behavior, genetics, and economics. For example, social analysis offers a system level approach in explaining how social disparities in health outcomes come into existence and how they are reinforced. Paul Farmer suggests that even in the context of a health condition which is very well characterized on a biological-level, its emergence, course and outcome is shaped by "social forces" such as racism, pollution, poverty, infrastructure or policies; therefore health phenomena are "biosocial" and not entirely biological in nature (Farmer et al. 2006). Social disparities in health are also "structural" 
because social forces influencing health emerge from the political and economic context and decision-making processes. Therefore, we have to acknowledge that experiences affecting function and structure of the brain across the life-course emerge from a social world.

According to the ecological systems theory, there are two major social structures which influence a developing child (Bronfenbrenner in (Boyden, Dercon \& Singh 2014)). On the one hand, the family and/or peer group constitute a micro-system directly influencing child development via frequent interactions and activities. The micro-system, apart from material conditions, provides a developing child with specific values and meanings which shape the child's perception of the environment. This is important because the perceived, and not the actual objective environment, might be the key in early childhood development. On the other hand, this micro-system is shaped by the macro-system referring to broader political-economic and socio-cultural contexts. Therefore the nature of early childhood development is determined partly by potentially modifiable environmental factors on the micro-system level and the opportunities provided by the pervading economic conditions and socio-cultural constraints on the macro-system level. However, the scientific community across multiple fields has acknowledged that direct care-givers within a family have the greatest influence on child cognitive and socio-emotional development (Heckman 2006).

The concepts of socio-economic status (SES) and socio-cultural capital are of special relevance in the context of the micro- and macro- systems envisioned by Bronfenbrenner. On the one hand, SES is defined on the level of an individual ("micro-system") and has been associated with multiple health outcomes via cognitive and behavioral pathways. The effects of SES might be mediated via material resources or specific practices characteristic to a particular socio-economic stratum. More directly, not being able to afford high quality food might result in child's undernutrition/stunting leading to deficits in neurodevelopment. Indirectly, within low SES families, caretakers might be pre-occupied with multiple responsibilities, therefore have less opportunities to spend high quality time with a child and providing him or her with socio-cognitive stimulation. It is worth noting that SES is dynamic across the life-span depending on the degree of social and economic risks and opportunities. This is important because even a brief socio-economic shock experienced by a household might have a profound impact on early childhood development (in (Boyden, Dercon \& Singh 2014)). On the other hand, social capital is usually characterized by the nature of the availability of economic as well as human, cultural resources or social interactions (Almedom \& Glandon in Kawachi et al. (eds), 2008:191). It acknowledges the fact that as social individuals we do not only live within a border of a household but are constantly influenced by a greater community ("macro-system") and socio-cultural context that varies in form and complexity across developed and developing countries. In theory, higher levels of social capital can compensate for lower SES and thus constitute another level for intervention strategies. Overall, the complexity of the social and culturally complex world that give rise to the variety of experiences has to be considered while thinking about possible interventions targeting disparities in brain outcomes.

Social determinants in brain outcomes are extremely complex. The concept of SES has been extensively examined in the field of health sciences. However, the question of how SES impacts specifically brain structure and function is a relatively new initiative within the field of brain sciences. The idea behind this paradigm is that SES might act directly on brain structures and/or function or it can be mediated by specific life experiences and behaviors associated with SES (Brito, Noble 2014). Studying separate measures of socioeconomic status might be difficult because of close relationship (correlation) between such factors. In the field of cognitive neurosciences, SES has been described and measured in multiple ways: household or family income, parental education or educational attainment, parental occupation, neighborhood SES or SES composite measures. Importantly, in all these cases, SES is just a proxy for the collection of positive and negative life experiences impacting brain structure and function termed "experiential correlates of SES" (Brito, Noble 2014). 


\section{PART II - SKILLS AND THE BRAIN}

\section{NEURAL CONNECTIONS AS THE FOUNDATION FOR SKILLS FORMATION}

The brain, although to a lesser extent, remains malleable across the life-course. Although details regarding adult plasticity extends beyond the scope of this paper, it is important to highlight some of the most important forms of adult plasticity to understand that neuroplasticity might "interfere" with brain structures and functions in the developing and aging brain.

Synaptic plasticity is one of the experience-induced changes underlying learning and memory and thus skills formation. The acquisition of new knowledge, retention and storage of new information rely on the weakening and strengthening of synaptic connections which are essential for skills development. Although the brain continue to form synapses throughout the lifetime, the processes governing changes in synaptic connections in adulthood differ from the ones that occur during early brain development (Kolb, Gibb 2011). Later in life, the synaptic changes are induced to specific experiences and are more local - therefore those synapses are not "experienceexpectant" but more "experience-dependent" (see Box 3). The common feature of synaptic plasticity in early and later brain development is the requirement of high quality and repeated experience for an optimal outcome. During an experiential learning activity, different neurons from different parts of the brain talk to each other and fire signals at different time points. Once the activity is repeated and neurons fire together in a synchronized fashion, according to the Hebb's Rule "neurons that fire together, wire together" - the molecular knowledge about the activity is consolidated and easier to extract next time the same activity is performed.

Other forms of plasticity are at work in later-life. For example, glial cells are born just after most neurogenesis is complete and continues throughout life (Kolb, Gibb 2011). Also, the process of myelination continues through the $3^{\text {rd }}$ decade of life. Multiple studies indicate that new neurons are formed (neurogenesis) in certain areas of the brain even in adulthood, for example in the region of hippocampus. One of the most influential studies in the field of adult brain plasticity (Maguire et al. 2000) indicated that experienced taxi drivers in London, a city that requires high levels of navigation abilities, had regionally enlarged hippocampi in comparison to controls who did not drive taxis. Furthermore, regional hippocampal volumes correlated with time spent as a taxi driver. Although the results were prone to reverse causation bias, the study indicates the possibility of substantial training-induced brain plasticity occurring in mid-life with positive consequences on brain structure and function.

\section{COGNITIVE AND SOCIO-EMOTIONAL FUNCTIONS AND SKILLS}

One of the ultimate goals for successful brain development is to ensure that a child develops highlevel cognitive processes to tackle novel problems and reason about the world. In addition to the attainment of cognitive functioning, another ultimate goal is to ensure that a child acquires highlevel socio-emotional skills to function well in socially challenging environments. Brain complexity programmed in early life directs the development of human abilities. The activity of neurons in the brain gives rise to cognitive, socio-emotional and executive functions underlying skills development. Cognitive events involve processes such as reasoning, processing speed, memory and spatial ability (Deary, Penke \& Johnson 2010) as well as language (Pessoa 2008). The more nebulous concept of emotion refers to states such as anger, fear or happiness. Brain morphology and cognitive as well as socio-emotional functioning change across the life-span.

There are two main aspects of cognitive ability: crystallized and fluid. Crystallized ability, or knowledge-based ability, is defined as "the individual's store of knowledge about the nature of the world and learned operations such as arithmetical ones which can be drawn on in solving problems" (Nisbett et al. 2012). Cognitive capacities such as logical thinking, speed of thought and problem solving ability, aspects of memory and cognitive control are contained within the term of fluid 
abilities. They tend to correlate (individuals who perform well in one domain have a tendency to perform well in the other domains) and are closely linked to general cognitive ability (Deary, Penke \& Johnson 2010) which depends on the integrity of the entire brain (Deary et al. 2009). Since fluid and crystalized abilities have different trajectories across the life-span, it is believed that they are underlined by distinct cognitive functions.

Cognitive and socio-emotional skills give rise to higher order brain processes which underline complex skills important for future life success. A system called "Executive Functions" (Cognitive Control) acts as the umbrella for both cognitive and socio-emotional processing (Naudeau et al. 2011). It is the brain's capacity to consciously control our thoughts and actions to plan and execute goals. Due to improvements in mental processes controlled by Executive Functions, a child becomes better at organizing thoughts, working towards long-term goals, ignoring irrelevant information, and controlling impulses (Silvia A. Bunge, Allyson P. Mackey, and Kirstie J. Whitaker 2009).

Both Executive Functions and fluid cognition rely on working memory - a central component of human cognition. Working memory contains sensory or memory-like information over a short period of time that we can act on and process. It is often represented as a "mental blackboard" which keeps relevant information "online" in order to carry out an immediate goal (Silvia A. Bunge, Allyson P. Mackey, and Kirstie J. Whitaker 2009). For example, when we are introduced to a new colleague, our ability to recall the colleague's name minutes later depend on bringing that information back into working memory for further processing. The ability to retain and manipulate information improves over childhood development and is an important feature of school readiness and academic success.

\section{COMPLEMENTARITIES BETWEEN BRAIN FUNCTIONS IN SKILLS FORMATION}

Multiple policy reports concerned with early childhood development present cognitive and socio-emotional skills as a rather dichotomous construct in which one set of functions acts in opposition to the other (thus the term "cognitive" versus "non-cognitive" skills). Some tend to divide brain functions and regions into more 'affective' or 'cognitive' but increasing evidence from brain sciences provides us with more clues into why "cognitive" and "non-cognitive" or rather "socioemotional" functions are interdependent. As Pessoa points out, "complex cognitive-emotional behaviors have their basis in dynamic coalitions of networks of brain areas, none of which should be conceptualized as specifically affective or cognitive" (Pessoa 2008).

The concept of the cognitive and emotional brain divide is not scientifically proven (OkonSinger et al. 2015). In fact, the more emotional brain regions are also involved in cognition; the more cognitive brain regions are also involved in emotions; finally, cognitive and emotional pathways in the brain are integrated and they jointly contribute to behaviors (Pessoa 2008). Cognition and emotions are governed by a number of overlapping neural systems (Phelps, Elizabeth A. and Delgado, Mauricio R. 2009); both cognitive and socio-emotional processes share common antecedents and underlying physiology in development (Richards, Hatch 2011). On the one hand, cognitive events engage cortical regions (Pessoa 2008), trigger behavior and are the consequence of parallel processing whereby different modules of the brain influence activity in other modules in countless ways (Gazzaniga, Doron \& Funk 2009). On the other hand, emotions, often represented as irrational and spontaneous processes governed by more "primitive" brain structures (such as the amygdala and hypothalamus (Pessoa 2008)), play a crucial role in emotional learning and memory as well as social interactions. Importantly, brain areas with a high degree of connectivity, called hubs, regulate the flow and the integration of information between the "emotional brain" and the "cognitive brain"; this highly dynamic and context-dependent process is thought to reflect the cognitive-emotional interactions (Pessoa 2008). For example, amygdala, a region of the brain that was thought to be purely associated with emotional functions, has been recently identified as a cognitive-emotional connector hub (Young and colleagues in (Pessoa 2008)). 
Certain functions are not limited to a single brain area or structure but rather rely on both cortical and subcortical components and complex neural networks between them. For example, different regions of prefrontal cortex (PFC) control the more - "cognitive" and more- "socioemotional" neural processes. More specifically, cognitive and socio-emotional functions rely on the maintenance of neural activity in PFC which coordinates the activity of billions of neurons extending over much of the brain (Miller, Cohen 2001). This pattern of neural activity involving higher and lower level processes represents the formulation and achievement of goals which gives rise to purposeful and goal-oriented behaviors, the key feature of Executive Functions (Cognitive Control). Finally, studies suggest that the fine-tuning of neural connections within prefrontal and parietal areas of the cortex and increased neuronal connectivity within and between these and other more "superficial" regions are likely to underlie improvements in working memory, Executive Functions as well as fluid cognition during development (Silvia A. Bunge, Allyson P. Mackey, and Kirstie J. Whitaker 2009). Therefore evidence based on structural and functional features of the developing brain confirms the complexity and interrelationship of cognitive and socio-emotional processes. The different regions and structures of the brain are involved in cognitive and socio-emotional functions which give rise to cognitive and socio-emotional skills underlying complex behaviors.

\section{BRAIN'S FUNCTIONAL DEVELOPMENT}

Figure 3: Development of Neural Networks

Neural Network The brain begins developing in the womb and achieves dramatic levels of growth during the first few years of life. During this time positive experiences contribute to building a strong brain architecture.

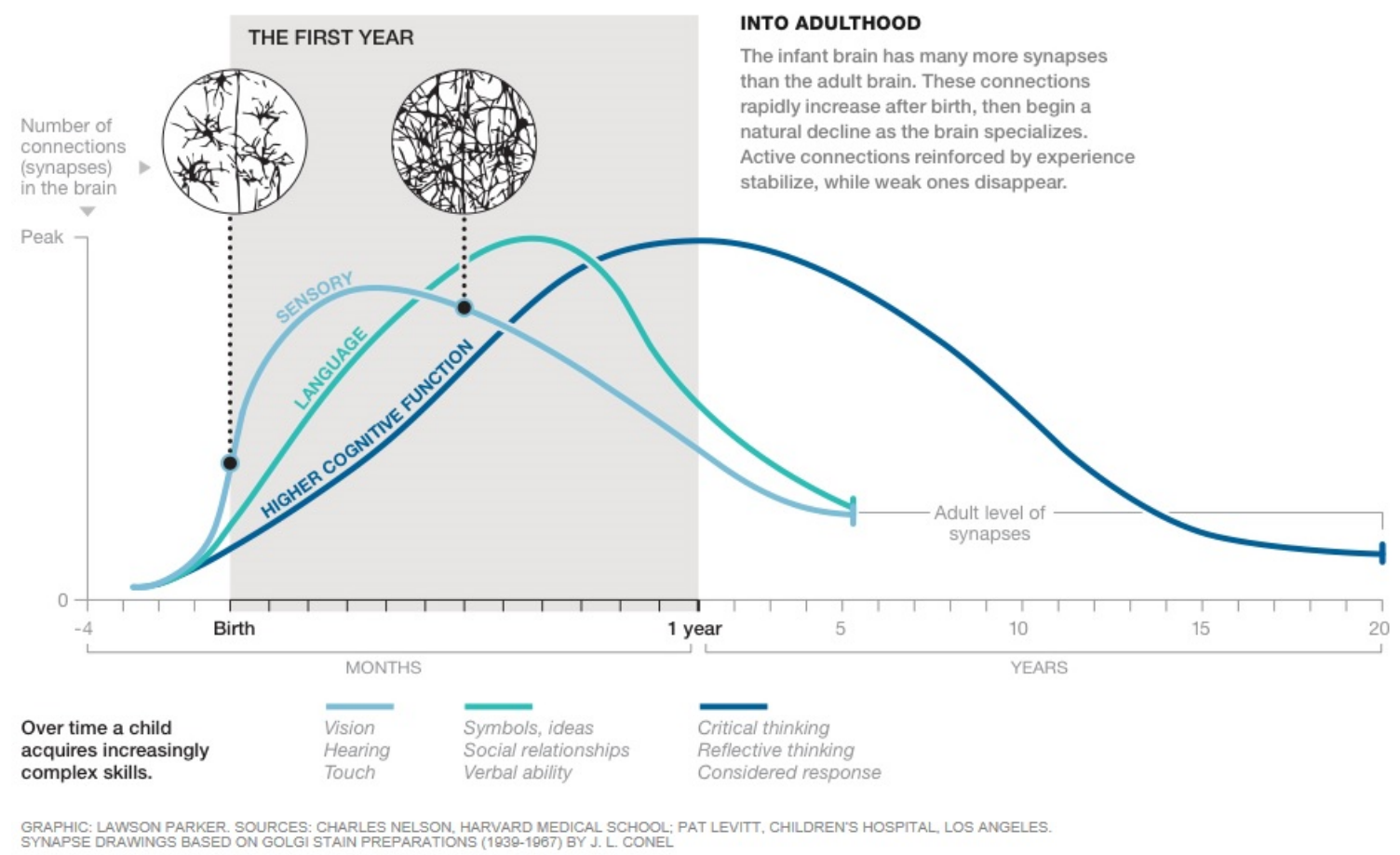

Source: in http://ngm.nationalgeographic.com/2015/01/baby-brains/neural-network-graphic

Different areas of the brain mature at different rates (Figure 3). As already mentioned, the overproduction of synapses for a particular faculty is achieved at different time points and is followed by the loss of unnecessary connections. Areas controlling sensory processing mature first. The lower adult levels of neural connections in the areas of the brain that control vision, audition and language are achieved by age 5-6. In contrast, while child's sensory cortex develops its full functionality in the first few years of life (Mackey et al. 2012), the brain areas supporting complex cognitive and socio-emotional functions mature last by age 18 (Thompson, Nelson 2001). For 
example, PCA controlling important skills such as reasoning, problem solving, self-regulation, personality, and social functioning achieves its full maturity beyond the $2^{\text {nd }}$ decade of life (Mackey et al. 2012). Since developmental period for PCA is substantially prolonged, this makes this brain area especially vulnerable to environmental insults or, on the more positive side, malleable to positive experiences for longer (Mackey et al. 2012).

\section{BRAIN FUNCTIONS AND THE AGING BRAIN}

It is challenging to distinguish between normal and abnormal cognitive aging and there are substantial individual differences of cognition across the life-span (Deary et al. 2009). However, on average knowledge-based abilities remain relatively stable into late-life and fluid abilities decline with advancing age starting in mid-life contributing to age-associated cognitive decline (Nisbett et al. 2012, Mustafa et al. 2012) due to age-associated brain pathology. The prevention of decline in 'fluid' abilities is important for multiple reasons (Deary et al. 2009). First, 'fluid' domains are essential for every-day activities, living independently and general well-being. Second, declines in speed of information processing drives the overall age-associated cognitive decline. As pointed out previously, the density of neural connections in the frontal cortex drops with increasing age (Small, Mok \& Bornstein 2001). Unsurprisingly, since PFC underlines the cognitive control system which supports goal-oriented behaviors, impaired executive functions (in addition to mental speed) have been proposed as the mediator of the overall age-associated cognitive decline - emphasizing the importance of healthy PFC and executive functions across the lifespan (Whalley et al. 2004).

\section{Box 4: "Early-life environment determines brain development and brain aging"}

There is a growing body of evidence that brain complexity programmed in early life facilitates cognitive and socio-emotional maturation, retention of brain functioning across the lifespan and healthy brain aging. Optimal bran development will provide an individual with a greater number of neurons, more synapses and multiple pathways for any given task. Such "neuronal redundancy" comes in handy when faced with deleterious brain aging. Within the context of cognitive aging there is suggestive evidence that the peak level of fluid cognitive abilities, shaped in part by (early) childhood cognition - which itself has been affected by exposure to stimulation, nutrition, and stress in early-life - is one of the major factors in determining cognitive aging trajectories.

Childhood cognitive functions provide a basis for adult cognitive functions which remains fairly stable across the life-span (i.e. the rank is preserved) (Deary, Whiteman, Starr, Whalley, \& Fox, 2004). In fact, early childhood cognitive ability is a very powerful predictor of later-life cognitive functions and the results are remarkable: it explains more than $50 \%$ of the variance in mid-life and late-life fluid cognitive abilities (Deary, Whalley, Lemmon, Crawford, \& Starr, 2000). The heritability of general cognitive ability, closely associated with fluid abilities, is estimated at $\sim 50 \%$ and because the brain is the most sensitive to environmental influences in very young childhood, this proportion increases over the life-course to even 80\% in later-life (Deary in Richards, 2012).

An increasing number of studies have suggested that early childhood interventions targeting mental domains might increase maximum life-time cognition, potentially reduce the trajectory of cognitive decline in late-life, and postpone the point at which cognitive deficits appear. One of the studies concluded that 'ethnic and socioeconomic disparities in cognitive function in older Americans arise primarily from differences in 'peak cognitive performance' achieved earlier in the life course and less from declines in later life' (Karlamangla et al., 2009). Although multiple complex pathways have been proposed to explain this association (for example, childhood cognitive abilities might provide, partly via education, entry to better jobs and healthier environments), optimal early-life environment (Staff et al., 2012) and brain developed to its full potential (Richards \& Sacker, 2003) might play the key role. 
It should be clear by now that our ability to exercise certain skills depends importantly on our brains: the building blocks as well as the underlying cognitive and socio-emotional processes within our brains.

\section{EARLY-LIFE SOCIO-ECONOMIC DISPARITIES IN BRAIN FUNCTIONS}

Education, sociology, public health and anthropology have already provided us with tremendous knowledge on understanding the links between SES and childhood development in terms of cognitive and educational achievements (Noble, Farah 2013) and an increasing number of studies within brain science contribute to this knowledge. Multiple studies from low-, middle- and highincome countries have shown that early childhood conditions as estimated by socio-economic status (SES), facets of poverty, or more specific elements of SES are associated with cognitive functions across the life-span, staring in early childhood. The impact of economic shocks on cognitive functions is causal and well-established. It appears that children from more wealthy families perform better on cognitive tests than children from poorer families creating what is called socio-economic disparities of individual differences in brain functioning across the life-course.

Early-life socio-economic disparities affect multiple brain functions that are important for school readiness skills. For example, Noble et al. has shown that these socioeconomic gradients are present for specific brain functions including language, memory and executive functions with language being affected the most (Noble, McCandliss \& Farah 2007). Importantly, socioeconomic disparities in developmental trajectories of language and memory are already detected before two years of age (Noble et al. 2015); disparities in more specific aspects of language such as vocabulary and language processing efficiency are already evident at 18 months (Fernald 2013) (Fernald, Marchman \& Weisleder 2013). In both studies home environment and the characteristics of parenting practices played an important role in mediating the socio-economic disparities in brain functions. In the former study, these were the home language/literacy environment and parental warmth that in part explained these differences (Noble et al. 2015); in the latter one, it was richer language experience (Fernald, Marchman \& Weisleder 2013). This evidence is supported by quite shocking results indicating that there is a 30 million word gap by age 3 in the USA between children of well-educated families and children from the families on welfare (Hart 2003). Therefore these results indicate that the socioeconomic disparities in brain functioning appear relatively early. Also, they point out to the importance of supporting and nurturing environments provided by caregivers on optimal brain development.

Similar early-life socioeconomic gradients in the same brain domains are present in lowand middle- income countries. Fernald et al. found that in a large nationally representative sample of children in Madagascar, children whose families were in the top wealth quintile or whose mothers had secondary education performed better on almost all cognitive and language tests compared with children whose mothers were in the lowest wealth quintile or with no education (Fernald et al. 2011). The mean difference in scores doubled between ages 3 and 6 years; the disparities were the greatest for language and executive functions components including working memory and sustained attention. In addition, based on multiple longitudinal studies from Kenya, Brazil, Guatemala, Philippines, South Africa and Indonesia, wealth at birth was consistently associated with later cognitive or academic performance within the age range 12 months - 18 years whereby poor children performed worse compared with children born into more affluent families with the effect sizes ranging from 0.70 to 1.24 SD scores between the top and bottom quintiles (Grantham-McGregor et al. 2007). Overall, these findings suggest that socioeconomic gradients in brain functioning during childhood exist across the socio-economic spectrum and even in the context of extreme poverty. The effects of poverty on the overall childhood development in middle and low income countries have been already summarized (Walker et al. 2007). 


\title{
PART III - POLICY LEVERS AND BRAIN DEVELOPMENT:
}

\author{
THE ROLE OF SOCIAL POLICIES IN SHAPING BRAIN FUNCTIONS AND \\ STRUCTURE
}

\author{
How do Socioeconomic and Psychosocial Influences "get under the Skull"?
}

Understanding the mechanisms of early-life socio-economic disparities in brain function is crucial for the design of effective and scalable early interventions. To eliminate the socioeconomic disparities in brain function, we need to understand the neural pathways of how early life-conditions (SES, poverty) or its facets i.e. specific modifiable factors such as nutrition or stimulation "get under the skull". With this knowledge, we will be better equipped to design and evaluate appropriate initiatives aimed at reducing life-course individual differences in brain functioning. Research on these questions is of high relevance to the global intervention community. Multiple professionals from the variety of fields who design and implement early childhood development interventions to improve brain outcomes have recognized the potential advantages associated with the introduction of biomarkers to identify the best timing and effectiveness of an intervention. This field is still in its infancy - only recently, with the advancement of neuroimaging and electrophysiology modalities and techniques, we are just beginning to understand the underlying neurobiological mechanisms and the potential neural pathways of these associations.

In order to come up with appropriate policies and interventions to eliminate the visible early-life socioeconomic disparities in brain outcomes, we need to know the answer to the following three questions: WHICH brain systems are important? WHEN are disparities in neural and cognitive development first detected?; and HOW can different potentially modifiable factors explain these differences? (Noble, Farah 2013). Another crucial question is: WHO should constitute the priority target group?

\section{WHICH Brain Systems are Important during Development}

Brain systems affected by early-life socioeconomic adversities play a critical role in cognitive domains important for children's school readiness skills. In an invited review coauthored by Brito and Noble (Brito, Noble 2014), the authors comprehensively summarized the state of the field examining the relationship between early-life socio-economic status (SES) and brain development with several key messages. First, different dimensions of early-life SES might reflect various life experiences and therefore differentially impact brain structures. Multiple aspects of SES in early-life have been shown to be associated with specific brain constructs in childhood and later in life as opposed to global brain differences - therefore SES disparities in brain outcomes are domain specific. These associations are in a negative direction such that "poorer" SES aspect is associated with greater neural deficits in multiple brain areas at different time-points (Pavlakis et al. 2015). These specific structure include PFC, the temporal lobe (memory and language comprehension), and the limbic circuitry involving amygdala and hippocampus. These findings are important because the identified structures are involved in relatively distinct brain circuits and support skills essential for later school achievements (Brito, Noble 2014) such as language skills, working memory, cognitive control, mental flexibility, self-regulation skills and an overall ability to form meaningful relationships. This is important because these individual behaviors form the basis for healthy and productive societies.

Overall, these comprehensive reviews and studies have indicated that early life adversities have an effect on brain structure and function across the life-span: children from more affluent families achieve greater structural and functional brain capacity and therefore better brain's potential in multiple domains. These circumstances put them at an advantage for the years to come: children of higher pre-school cognitive and socio-emotional abilities are more likely to perform well at school and therefore less likely to drop-out from school, gain a better and potentially more cognitively 
stimulating job, are better able to cope with stress, avoid a variety of risky behaviors, and select healthier lifestyles.

\section{WHEN are disparities in neural and cognitive development first detected}

Socioeconomic differences in neural development begin to emerge in infancy. Tomalski et al. have detected socioeconomic disparities in functional brain development driven by the frontal brain areas implicated in language and attentional processes in infants as young as 6- to 9-monthold! (Tomalski et al. 2013). In this study, with the use of electroencephalography (EEG) (see Box 5) the researchers recorded resting-state gamma-band activity in the infant participants who were presented with video clips representing toys and interacting faces. These differences were related to family income and maternal occupation but were not explained by the quality of infant sleep or parental education although could be potentially associated with the quality of home environment and early interactions with parents. These results support the studies indicating that language and executive functions development (in addition to memory) are most vulnerable to early-life suboptimal environments. Although there are only a few studies which investigated SES disparities in brain structure and function during infancy, the study by Tomalski et al. suggests that these differences might emerge and be detectable extremely early. Based on the studies reviewed in this document, a general consensus is that the sooner an intervention is provided the better for both functional and structural features of the brain (Nelson et al. 2007) (Bick, Nelson 2016). Similarly, the earlier the adversity impacts the brain, the more profound the disruptions to the brain, and lower the chances for recovery (Tomalski, Johnson 2010).

Because deficits in neural development may emerge early in infancy and early interventions can have a remediating effects on brain structure (Bick, Nelson 2016), reliable assessment and the early detection of brain developmental delays is crucial but challenging. Existing behavioral paradigms and the well-established assessment tools designed to measure cognitive and socioemotional functioning can only detect the impact of environmental factors (such as facets of poverty) once they reach the threshold for observable output (Lloyd-Fox et al. 2014). In addition, electrophysiology (along with neuroimagining) is a more culturally-neutral marker for brain outcomes and its adaptation as well as standardization is more straight-forward than for the more conventional tools, therefore offer role in informing targeted early intervention strategies (LloydFox et al. 2014).

\section{Box 5: Brain electrophysiology}

Electroencephalography (EEG) measures the electrical activity of the brain (Gazzaniga et al., 2009). Resting baseline EEG is commonly measured while infants are engaged in observation of general stimuli (Tomalski \& Johnson, 2010). EEG captures oscillatory activity at low (theta, alpha) and high (beta, gamma) frequencies - these oscillations are thought to reflect attentional processes, perceptual binding and maintaining high-level neural representations of objects. EEG is useful for studying infant functional brain development and for identifying early risk factors (Tomalski \& Johnson, 2010). In addition, resting EEG is cheap and relatively reproducible - therefore it could be utilized as a biomarker in assessment of various early interventions (Pavlakis et al., 2015). 


\section{WHO Should Constitute the Priority Target Group}

Noble et al. (2015) showed that family income is associated with total brain surface area among children from the poorest families. While most studies investigating the associations between SES and brain structure have measured cortical volumes (the product of cortical surface area and cortical thickness thickens), the team looked at the relationship between SES and cortical surface and cortical thickness separately, in addition to hippocampal and amygdala volumes, in a cohort of 1,099 typically developing individuals between 3 and 20 years of age (Noble et al. 2015). This novel approach recognizes that cortical surface area and cortical thickness have different developmental trajectories. In this fascinating study, Noble et al. found that family income and parental education are associated with brain structures in regions critical for the development of language, executive functions and memory after controlling for genetic ancestry. Firstly, an additional time of a parent spent in education was associated with an increase in child's or an adolescent total brain surface area. Secondly, family income was associated with total brain surface area such that for every dollar increase in income, the increase in brain surface area was most detectable among the poorest families. In addition, children's whole brain surface area, but not cortical thickness, indirectly mediated the links between family income and executive functions. Possibly these associations have their origins in prenatal or postnatal environmental disparities; however, the researchers were not able to test this hypothesis with the available data. The fact that the total brain surface area, but not the thickness, mediates the link between SES and executive functions is important; it is believed that cortical surface area is shaped by experience-related synaptic pruning whereas cortical thickness reflects the more experience-expectant type of synaptic pruning (Noble, Houston et al., 2015) - pointing to the possibility that the link between income and the brain functions examined in the study are mediated by brain processes malleable to experience and therefore can be potential targets for interventions. However, the authors of this single study did not elaborate on this issue further emphasizing that these novel findings based on a cross-sectional design require validation. Overall, these results imply that early childhood interventions might have the greatest impact among the most disadvantaged families.

Hair et al. (2015) found that the influence of early-life low socioeconomic circumstances on cognitive and academic performance across the life-course is mediated by structural brain development. This very recent brain imaging study presented "even more powerful evidence" (Luby 2015) confirming the relationship between low socioeconomic status and impaired brain structure. Importantly, Hair et al. extended the idea further to determine whether the detected changes in structural brain development mediate the relationship between household poverty and poorer academic performance (Hair et al. 2015). In this study, 389 typically developing children and adolescents aged 4 to 22 years underwent longitudinal magnetic resonance imaging (MRI) scans (see Box 6, (Murray 2012)) and detailed cognitive and academic achievements assessments. Household poverty was measured by family income adjusted for family size as a percentage of the federal poverty level. In this sample, children from poor households scored on average $4-7$ points lower on standardized academic tests. Importantly, regional gray matter volumes in the frontal lobe, temporal lobe and hippocampus were significantly lower in low-income children relative to the developmental norms; the largest gaps were detected in children below the federal poverty line. It has been estimated that $20 \%$ of the deficits in test scores could be explained by structural differences in the frontal and temporal lobes. The major strengths of the study were communitybased sampling strategy so that the children reflected regional and overall US demographics and strict exclusion criteria to study typical brain development therefore the effects were likely underestimated. Another advantage was the longitudinal design and statistical modelling which accounted for multiple observations within a single participant. It is important to point out that similarly to the study performed by Tomalski et al., in this sample poor families were highly educated and the effects were not explained by differences in early health or parental education suggesting that the accumulation of circumstances associated with poverty can have a profound and direct impact on the developing brain. Taken together, these data are consistent with the notion that those from the poorest households, who will have greatest brain maturational lags, need the early life investment most. 
Recent work supports the idea that children from disadvantaged backgrounds, in addition to having brain developmental delays, acquire more brain burden associated with aging. Staff et al. examined the effects of childhood socioeconomic status on adult brain size and its components among members of the 1936 Aberdeen Birth Cohort aged 68 years (Staff et al. 2012). To investigate this association, Staff and colleagues used structural equation modeling and volumetric magnetic resonance imaging (MRI) which quantifies volumes of brain structures (see Box 6). They demonstrated a significant association between childhood SES as index by paternal occupation at age 11 and hippocampal volumes in older adults without dementia - older adults with low SES in childhood had smaller hippocampi. These effects were present after taking account for life-course variables such as cognitive ability at age 11 years, adult socioeconomic status, gender, and education. Thus, this study extends the current findings to the possibility that early-life socioeconomic disadvantage impacting structural brain development might endure for more than 50 years. Simultaneously, Staff et al. did not find any association between childhood SES and whole brain volume confirming earlier results that early-life environment affects specific brain structures as opposed to the whole brain.

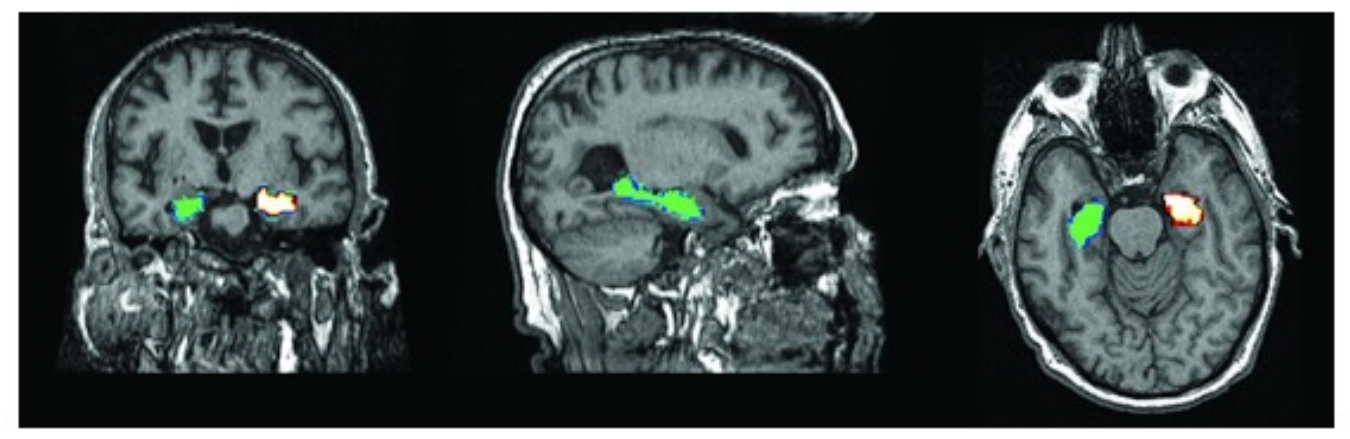

A.D. Murray AJNR Am J Neuroradiol 2012;33:1836-1844; @2012 by American Society of Neuroradiology

\section{Box 6: Brain Imaging I}

Magnetic resonance imaging (MRI) exploits the magnetic properties of the organic tissue of the brain to image its structure (Gazzaniga et al., 2009).

Here the assessment of hippocampal volume loss on structural MRI; automated voxel-based quantification of hippocampal volumes by using FreeSurfer, showing left and right hippocampi (Murray, 2012). 
A second study performed within the same cohort (Murray et al. 2014) indicated that early life socioeconomic disadvantage is associated with increased brain imaging evidence of hyperintensity burden (cerebrovascular disease) in late-life, with its established negative consequences for cognition, stroke, dementia and survival (see Box 7). The team detected an obvious cut off between "blue collar" and "white collar" paternal occupations in increased white matter hyperintensity burden. The major strength of both studies lies in the availability of childhood intelligence rarely available for aging cohorts. Together these results indicate that childhood disadvantage poses a "double whammy" of brain pathology and provide insights into the disparity in cognitive outcomes across the life span as well as dementia prevalence in those from different socioeconomic backgrounds. The mechanisms underlying these effects are unknown, but may act through fetal and/or early life programming in line with the Barker's hypothesis (for additional information, see Box 11). In summary, those from the most disadvantaged backgrounds in childhood (Noble et al. 2015), who will have greatest disease burden in late-life (Murray et al. 2014), need the investment most (Hair et al. 2015).

\section{Box 7: Brain Imaging II}

Magnetic resonance imaging (MRI) exploits the magnetic properties of the organic tissue of the brain to image its structure (Gazzaniga et al., 2009).

Here the assessment of white matter hyperintensities (WMH) measured by using semiquantitative visual rating scales for the evaluation of signal hyperintensities on MRI (Murray, 2012).

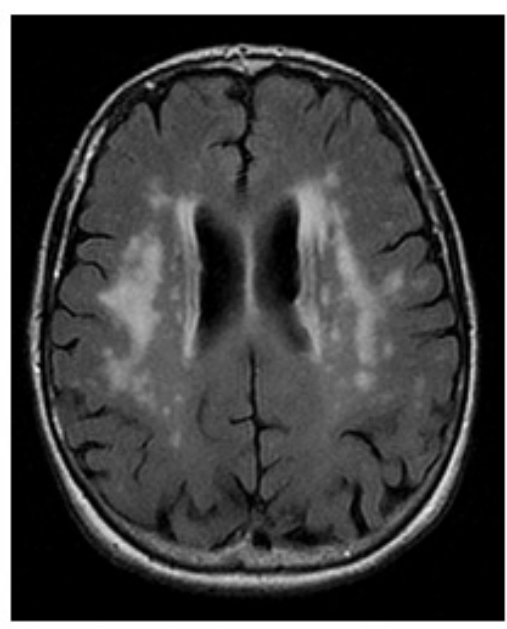

Figure courtesy of Dr. Chris McNeil, Aberdeen Biomedical Imaging Centre, University of Aberdeen, UK 


\section{BRAINS RESILIENCE AT THE CORE OF SOCIAL POLICIES}

\section{Recovery in Brain Structure and Function}

The brain's recovery from early-life insults is possible even when children experience severe deprivation during early development. In 2000 a new study, the Bucharest Early Intervention Project, investigated the consequences of early-life institutionalization characterized by limited access to language and cognitive stimulation, and inadequate caregiving (Bick et al. 2015) on brain development (Zeanah et al., 2003). It is the only existing randomized controlled trial of foster care for institutionalized children in Romania. As part of this study, 136 infants aged around 2 years at recruitment were randomly assigned to remain in the institution or to move to foster care - family homes which could be a beneficial alternative to the institutions due to better caregiving environment. A community comparison group consisting of 72 children who were never institutionalized was also recruited. A proportion of children $(n=69)$ at 8 years of age underwent structural (brain) imaging MRI scans which measures volumes of brain structures (see Box 6), here gray and white matter.

In one of the very recent study projects, Sheridan et al. examined the potential for recovery in total grey matter and white matter volumes among children who were initially institutionalized and then randomly assigned to foster care (Sheridan et al. 2012). First, both institutionalized and foster care children had significantly lower global gray matter volumes compared to the community control group - children who were never institutionalized. Second, children who remained in the institution had significantly lower total white matter content relative to the community comparison group. However, there was no difference in white matter volumes between children who were randomized to foster care homes and children who were never institutionalized. This increase in white matter (essential for cognitive and emotional development) among the intervention group was interpreted by the authors as the brain's potential for recovery: 'the potential for developmental 'catch-up' in white matter growth, even following extreme deprivation" (Sheridan et al. 2012).

After this finding, the follow-up study examined the potential long term improvements in white matter microstructural integrity - more specific feature of white matter pathways measured with diffusion tensor imaging technique (DTI) (Bick et al. 2015) (see Box 8). Alternations in microstructural integrity of white matter tracks involved in Executive Functions, emotional functioning and learning as well as sensory processing were associated with early-life neglect. Again, children who were randomized to foster care homes did not differ from children who were never institutionalized with respect to the white matter microstructure while children who remained institutionalized showed marked alternations in the integrity of the white matter circuits. 


\section{Box 8: Brain Imaging III}

Diffusion Tensor Imaging (DTI), performed with magnetic resonance scanners, is used to measure white matter pathways (the axon tracks) in the brain (Gazzaniga et al., 2009).

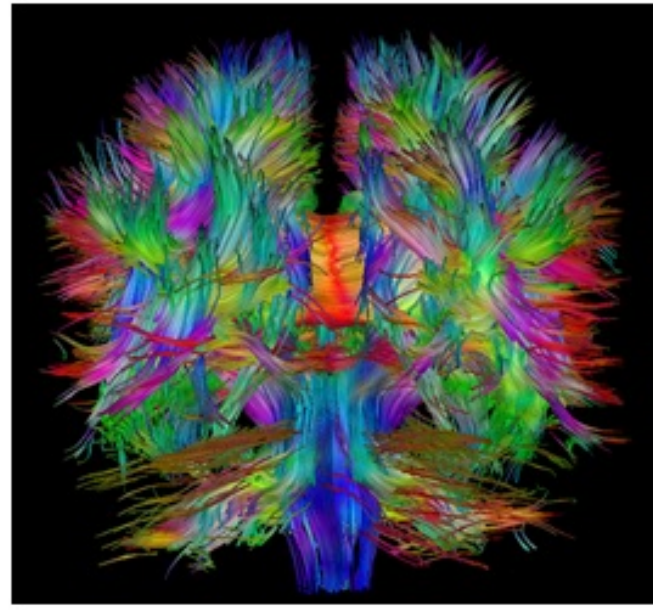

Figure courtesy of Dr. Gordon Waiter, Aberdeen Biomedical Imaging Centre, University of Aberdeen, UK

Even though the results of both studies cannot be generalizable to a general children population, they indicate that the brain is capable of developing resilience mechanisms to severe early-life neglect if provided with the opportunity to continue development in caring and enriching environments. In other words, the developing and highly malleable brains of children who initially experienced extensive psycho-social deprivation and then were moved to more stimulating and nurturing caregiving environments were able to 'catch-up'. Even though large socioeconomic gradients have been identified in language, memory and executive functions (Noble, McCandliss \& Farah 2007) further longitudinal studies need to determine which brain functions can be recovered later in life and which are the ones that are permanently marked by severe deprivations if the sensitive period is missed. Although mechanisms of brain recovery upon early interventions are complex, there is "clear evidence" that protective early-life factors (or removal of risk factors) can positively impact the brain and ameliorate adverse outcomes (Bick et al. 2015). Overall, the above studies emphasize again the importance of the high-quality family and caregiving environments during early childhood development. Also, as suggested by Noble et al. (2015), if this evidence based on correlational results is in fact causal, those from the most disadvantaged backgrounds (Noble et al. 2015), including children growing up in institutional settings, who will have potentially greatest brain developmental delays (Murray et al. 2014), need the early-life investment most (Hair et al. 2015).

\section{Harnessing the Power of the Human Brain}

Another unifying theme across early childhood development initiatives and brain aging is the notion of resilience, defined as the ability to withstand socially or biologically constructed adversity. In the field of cognitive neurosciences, the fascinating concept of cognitive reserve (CR) (Stern 2012) which requires quantification of neuropathology (e.g. with the use of imaging techniques (Murray et al. 2011)) implies that some individuals are able to remain cognitively healthy despite the accumulation of age-associated or dementia-related neuropathology. This is because structural or functional features of the brain allow individuals to overcome brain pathology due to the protective action of life-course factors such as early-life cognitive ability (which determines maximum life-time cognition, see Box 4 \& Figure 4), greater educational attainment, more complex occupations, and exposure to challenging mental activities with the former being a strong predictor of the latter three (Deary et al. 2009). These 
factors increase or maintain reserve in part because brain remains plastic and is capable of learning new things across the lifespan; and according to the "use it or lose it" principle, if you do not practice a domain, you are likely to lose the skill. A childhood cognitive ability is a major influence on educational achievements, work experience and opportunities to benefit from work-related training. These factors are potentially modifiable in early-life and, due to their accumulating impact over the life-course, may determine delayed onset of functional brain declines associated with aging.

Also, CR is in line with resilience to more socially grounded 'pathologies' such as economic, political or climatic crisis. In this context of resilience, the outcome is not necessarily a superior brain functioning but successful navigation of opportunities and individuals' investment in health or education despite the unfavorable social conditions. Furthermore, CR helps us to understand how a social phenomenon gets imbedded within human physiology which is essential for the design of effective interventions, especially the ones targeting early childhood development (Noble, Farah 2013). Finally, some very exciting studies indicate that people with strong motivational abilities (Maercker, Forstmeier 2012) (perhaps grittier individuals (Duckworth et al. 2007)) have greater resilience to cognitive decline and dementia (remember the complementary nature of cognition and emotions?). On an even greater micro-scale, neurons are capable of developing mechanisms that make them resilient to adversities in specific contexts (Double et al. 2010). Studies across the disciplines provide evidence that our neurons, brains and we as individuals and societies can function well despite social or even biological obstacles. Many of the qualities of brain resilience to biological and social factors are very likely shaped in early life. With the appropriate investments extending beyond early-life, resilience can be sustained across the life-course.

Finally, the resilient brain is at the core of "mind, society, and behavior" (World Bank Group 2014). With relevance to the current appreciation of behavioral sciences within the international development domain, the issue of early childhood development (ECD), aging, resilience, and skills development across the life-course is highly relevant to greater understanding of behavioral trajectories. How to maximize the so-called cognitive "bandwidth" (Mullainathan, Shafir 2014), i.e. cognitive potential supporting decision-making in the face of acute or systematic "scarcity", is the key question. Brain development across the life-course goes beyond the behavioral science toolkit and calls for neuroscience-informed interventions that would complement behaviorally-informed initiatives. This report encourages thinking about behavioral change from a developmental and lifecourse perspective by recognizing that decision-making processes across the life-course are rooted within the developing brain - therefore complements the discourse on neuroeconomics and decision making processes (Camerer, Loewenstein \& Prelec 2005). 


\section{POLICY INSTRUMENTS FROM A BRAIN DEVELOPMENT PERSPECTIVE}

\section{Box 9: HOW do Potentially Modifiable Factors Explain the Socioeconomic disparities in Brain Outcomes?}

We already know that early socio-economic disparities in multiple brain domains exist, and if no intervention is provided at the right point in time, they persist and even widen with increasing age. The availability of positive experiences help the brain grow and thrive across the life-course; the presence of negative experiences hurt brain development and impede a child's ability to flourish into a successful adult. The next question follows: what are some potentially modifiable mediators in the relationship between childhood SES and brain outcomes?

Lower socio-economic status and poverty might have a direct influence on brain function and structure or their effect might be mediated, for example, via inadequate food, poor sanitation and hygiene leading to increased infections and stunting. Noble et al. suggests that the associations between SES and brain structure could emerge from disparities in the prenatal environment as well as postnatal experience or exposures: (family) stress, nutrition, and cognitive stimulation. While details of how to intervene are beyond the scope of this report, below you can also find some examples of already implemented interventions. Because children in developing countries are exposed to multiple risks to brain development at once, it is very unlikely that a single-factor intervention will solve the issue of suboptimal brain development with its life-course consequences; rather, multi-factor interventions incorporating the features of behavioral change might produce the expected results (Aboud \& Yousafzai, 2015).

\section{Stress}

The brain is the key organ of vulnerability and resilience to stressful life events across the life-course. According to McEwen \& Gianaros, "stress-related processes [are] embedded within the social environment and embodied within the brain" (McEwen, Gianaros 2010). The brain determines what is threatening, regulates reactivity to physical and psychological stress and the underlying biological insults. Importantly, poverty and disadvantaged socio-economic circumstances lead to stress and this relationship is causal (Chemin, De Laat \& Haushofer 2013). Early SES-associated stress and its physiological consequences starting in utero has been implicated as a potential biological mechanism by which children from households of lower SES tend to perform poorly in comparison to children from households of higher SES. Importantly, children in low- and middle- income countries are especially at risk because the combination of socioeconomic, psychosocial and physical "stressors" in poor resource settings is multifactorial and very complex in nature. The brain mechanisms behind this association are just being discovered.

The field of health neuroscience brings us closer to the brain-dependent mechanisms associated with response to stress in face of socioeconomic circumstances. It is hypothesized that lower socioeconomic position associated with adverse and stressful environments as well as sociopsychological hardship (challenges of daily living, uncertainties of future prospects, and possibly social exclusion, marginalization and internalized states of demoralization) acts via the 
dysregulation of brain systems supporting emotional/social information processing and regulating functions associated with disease processes (Gianaros, Manuck 2010).

The biological mechanisms underpinning responses to prolonged so-called "toxic" stress in early childhood impairs the architecture of the developing brain with its life-long consequences (National 2014). Toxic stress is the most dangerous form of stress; it refers to stress responses whereby strong, frequent or prolonged sources of stress (e.g. child abuse or neglect, parental substance abuse, or maternal depression) are not balanced by supportive and adult relationships (Shonkoff et al. 2012). Within the life-course perspective, early-life stressors such as low childhood socioeconomic status and its facets may interact with genetic differences in abilities to affect the protective mechanisms of the limbic brain areas (McEwen, Gianaros 2010). Animal models have shown that even acute forms of prenatal stress experienced by the fetus (maternal stress) contribute to life-long dysfunctions in brain stress regulatory mechanisms involving hypothalamus (McEwen, Gianaros 2010). Therefore SES risk factors in early-life may determine vulnerability to stress even later in life; adult SES may interact with genetic individual difference to impact maturation and aging of the limbic brain areas.

It has been showed that amygdala controlled by prefrontal mechanisms and the activity of serotonin-releasing neurons (serotonin is a type of neurotransmitters) constitutes an essential "emotional" pathway in the embodiment of socioeconomic factors by the brain, especially in the context of stress responses dysregulation (Gianaros et al. 2008). For example, Kim and her colleagues examined the relationship between childhood poverty and neural activity during effortful attempts to regulate negative emotions (Kim et al. 2013). They showed that lower family income at age 9 was associated with reduced PFC activity and failure to suppress amygdala activation in young adulthood; this effect was mediated by the experience of chronic stress across childhood but not by the concurrent adult income. The detected association provides additional evidence for chronic stress mechanisms being involved in neural embedding of childhood poverty.

\section{Box 10: Stress - Examples of Interventions:}

The availability of positive experiences (protective factors) help the brain grow and thrive across the life-course; the presence of negative experiences (risk factors) hurt brain development and impede a child's ability to flourish into a successful adult. Therefore reducing stress reactions in young children is essential for optimal brain development (Denboba, Sayre \& Wodon 2014).

\section{Example 1:}

Exposure to violence has been identified as one of the priority risk factors for early childhood development in developing countries (Walker et al. 2011). Violenceprevention programs can reduce stress reactions in early childhood with the effect size from 0.56 to 0.91 (Walker et al. 2011). It has been shown that improving institutional environment of non-parental group residential care can lead to increased cognitive and socio-emotional skills in children (Denboba, Sayre \& Wodon 2014). 


\section{Nutrition}

The brain is "an energy expensive organ" - it accounts for 2 percent of an adult body weight but consumes 20 percent of the resting metabolism (Paus et al. 2010). Inadequate levels of energy supply in early years might reduce neural activity and the overall development (Aboud, Yousafzai 2015). This is why adequate, rich in micronutrients and balanced diet is essential for proper brain development. Child undernutrition can take different forms including stunting (low length or height for age), wasting (low weight for length or height), underweight (low weight for age) and deficiencies of micronutrients (vitamins and minerals) (Victora et al. 2010). Using the new World Health Organization (WHO) standards, Victora et al. (2010) described child growth patterns across 54 countries and concluded that early growth faltering of children in low- and middle-income countries is even faster and more alarming than currently assumed. The results brought to light new evidence because the WHO standards provided more precise references than previously (Victora et al. 2010). Multiple forms of child undernutrition have been associated with impaired brain outcomes across the life-course.

A third of children below age of 5 years in developing countries are stunned or have linear growth retardation (Walker et al. 2007). Stunting represents a range of biological and psychosocial risks and therefore has been identified as one of the indicators of poor development. Stunting, a form of growth failure, starts in utero or soon after birth; even though a catch-up growth might follow in the first two years of life, stunned children usually remain shorter for their age through to adulthood (Walker et al. 2007). Many prospective cohort studies indicate consistent and a robust finding of a significant relationship between stunting by age 2 or 3 and broadly defined childhood cognitive abilities, school enrollment/progress/attainment/performance after controlling for socio-economic covariates in low- and middle-income countries (Walker et al. 2007). A metaanalysis performed on longitudinal studies including the data from Philippines, Jamaica, Peru, Indonesia, Brazil and South Africa found that being moderately or severely stunted between ages 12 and 36 months (compared with not stunted) was associated with poorer cognitive functions with effect size ranging from 0.4 to 1.05 (Walker et al. 2007); stunting was also associated with grades attainment. In the context of socio-emotional development in young children, underweight and stunting have been correlated with apathy, less positive affect, lower levels of play, more insecure attachment, more problems with conduct and poorer social relationships at school age.

Intrauterine growth restriction in length is a major concern indicating a great need for nutrition interventions among pregnant women and women in childbearing age (Victora et al. 2010). Multiple studies have indicated that suboptimal intrauterine environments, such as nutritional deprivation in the womb, are associated with cognitive and socio-emotional dysfunctions in early-life. There is consistent evidence from middle- and low-income countries that intrauterine growth restriction, mainly due to poor maternal nutrition and infections in developing countries, is associated with developmental delays in early childhood (Walker et al. 2011). However, it is unclear whether the negative effects of suboptimal environment in utero on the developing brain persist into late-life and, to our knowledge, there are no studies from developing countries examining this relationship.

Multiple studies have indicated that maternal nutritional deprivation in pregnancy impacts the brain with long-lasting consequences. For example, studies framed as "tragic experiments of opportunity" have investigated the immediate and long-term impact of wartime famine on cognitive and socio-emotional functioning and its timing during gestation. The studies of individuals who were born at the time of the Dutch Famine during World War II $\left(^{*}\right)$ and whose mothers lived under 500 calories per day during pregnancy have shown that maternal starvation in early gestation is associated with cognitive dysfunctions and antisocial personality disorders in mid-life and in early adulthood respectively. Firstly, exposure to malnutrition in utero was associated with poorer attention performance - a fluid aspect of cognitive abilities prone to decline with advancing age. This relationship was also the strongest earlier in the pregnancy which is in accordance with the fact that the structure of the central nervous system forms at the start of gestation. The effect has been attributed to an accelerated cognitive aging process, although the effect of maternal stress 
rather than malnutrition per se cannot be ruled out (de Rooij et al. 2010). Such studies add to the validity and extension of the Barker's hypothetical framework (for additional information, see Box 11).

Box 11: The Barker's hypothesis has been suggested to account for the importance of the intrauterine environment on adult health.

According to Barker's hypothesis, in a low-nutrient intrauterine environment, the fetus adapts to these suboptimal conditions and programs its physiological and morphological states in anticipation of limited resources during development, a phenomenon called "phenotypic plasticity" (Barker, Eriksson, Forsen, \& Osmond, 2002). In case a developing fetus ends up growing up in the world of plenty (a common phenomenon in many developing countries undergoing economic and epidemiologic transitions), there is a mismatch between the programmed mechanisms and the actual environment; "compensatory growth" takes place at the cost of chronic conditions in later-life such as obesity, cardiovascular diseases and type 2 diabetes with transgenerational consequences (Barker et al., 2002). This phenomenon has been framed "The Developmental Origins of Health and Disease" (DOHaD). In fact, the hypothesis of "The Developmental Origins of Health and Disease" (DOHaD) has been just recently extended to the fields of brain development and mental health and therefore termed "The Developmental Origins of Behaviour, Health, and Disease" (DOBHaD) ((Van den Bergh, 2011) and Tuovinen et al. 2012) and awaits further studies on the developmental programming of neurodegenerative diseases (Giussani, 2011).

It is unclear whether the relationship between suboptimal birth outcomes and brain functions persists into late-life and, to our knowledge, there are no studies from developing countries examining birth effects into late-life. A handful of studies conducted in Europe, here UK and Finland, have shown respectively that 1 ) birth weight was associated with cognition in childhood and maintained across adolescence but was only weakly associated with cognitive functions at age 43 (verbal memory; speed and accuracy); those of lower birth weight were more likely to have lower educational qualifications and this relationship was partly accounted for cognitive abilities at age 8 (Richards et al. 2001); 2) those born late preterm had poorer episodic memory performance than those born at term. Importantly, among those with lower educational qualifications, late preterm birth was associated with lower performance on episodic memory, executive function, visual reproduction tasks, general neurocognitive abilities and a higher risk for mild cognitive impairment (MMSE-defined $\mathrm{MCl}$ ). Among those with higher educational qualifications, late preterm birth was not associated with neuropsychological tests. Collectively, late preterm birth might constitute a novel risk factor for neurocognitive impairment even in late adulthood and lifetime educational attainment may provide resilience to neurocognitive dysfunctions (Heinonen et al. 2015). More evidence is needed to establish the long-term and cumulative effects of suboptimal birth outcomes on cognitive and socio-emotional functioning across the life-course globally.

It is generally established that the window of opportunity to preventing undernutrition is contained within the first 1,000 days including pregnancy and the first 2 years of life (Victora et al. 2010). A recent systematic review and meta-analysis concluded that nutrition interventions with children ages 24 months and younger from developing countries have overall a relatively small effect on cognitive and language development (Aboud, Yousafzai 2015). As suggested by the authors, this overall effect might be attributed to several reasons; it is largely unknown: 1 ) whether macronutrients (carbohydrates, protein and fats) are necessary along with micronutrients; 2 ) what 
type and dose of micronutrients should be combined for best effects; 3) whether only deficient children should be subject to nutrition interventions. Potentially the solution is to integrate macronutrients, micronutrients, and nutrition education within the interventions. Nutrition education by itself is not likely to solve the issue in poor areas with high malnutrition (Aboud, Yousafzai 2015). More research is required to identify what nutrition interventions are most effective and how to ensure that investments in early-life nutrition persist into later-life.

A number of recent high quality studies highlight the detrimental effects of early malnutrition on later-life outcomes. New evidence includes reduced likelihood of formal employment at age 20-22 in the Philippines and lower psychological functioning in adolescence in Jamaica (Walker et al. 2011). Two recent analyses based on the Barbados Nutrition Study (BNS) showed that an episode of moderate to severe malnutrition within the first year of life results in neuropsychological deficits and impaired IQ in mid-life (Waber et al. 2014). This was the case even among individuals of normal birth weight who had achieved complete catch-up growth and had been subject to nutritional rehabilitation, emphasizing the critical role of adequate nutrition in the first months. Importantly, a recent and comprehensive review investigating the long term effects of stunting in childhood concluded that nutrition interventions implemented early in life in developing countries might have beneficial and sustained effects on brain outcomes provided that nutrition interventions are in place throughout and beyond the first 1,000 days (Tanner, Candland \& Odden 2015).

\section{Box 12: Nutrition - Examples of Interventions:}

Example 1: IN BANGLADESH, 61 million children suffer from malnutrition. Malnutrition leads to child stunting and impaired brain development. About two fifths of the children younger than five years old have stunted growth; about half of the two-year-olds are well below international height standards and about a third are severely underweight. Most Bangladeshi children do not receive early stimulation and learning opportunities. In this challenging context, researchers are studying the impact of a low-cost program that combines nutrition and stimulation for children under the age of three and their mothers. The results of this intervention will add to the growing body of evidence on how to most effectively improve children's physical and brain development. http://www.worldbank.org/en/programs/sief-trust-fund/brief/building-parental-capacityto-help-child-nutrition-and-health 
The assessment of brain structure and functioning along with behavioral measures to evaluate development might bring us closer to the question of when and how to design an effective nutrition intervention (Aboud, Yousafzai 2015). So far there are only few studies on neuroimaging and electrophysiological correlates of undernutrition in early life that could bring us closer to the identification of implicated neural pathways and more exact timing for optimal interventions. For example, in a very interesting study of high-school graduates in Chile aged 18 years from a low socioeconomic stratum, the group who had experienced severe undernutrition during the first year of life was compared to the group with no past history of undernutrition (Ivanovic et al. 2000). The main outcomes of interests included brain structure quantified with the use of MRI imaging (see Box 5), verbal \& non-verbal IQ, and school achievement (Spanish language and mathematics tests). The results indicated that the severely undernourished group had significantly lower birth weight, head circumference, IQ scores as well as aptitude test scores on all domains compared with the control group. They also had significantly lower absolute brain volume but the group differences within the corpus callosum subdomains, although lower in the disadvantaged group, were mostly insignificant. The study was based on a very small sample size and was to some extent exploratory in nature. However, it provides us with some insights into adolescence brain's structural and cognitive disparities according to nutritional status in the first year of life in already socio-economically disadvantaged high-school graduates.

Importantly, recent advances in an imaging technique called functional near infrared spectroscopy (fNIRS) (see Box 13) provide new capabilities for studying longitudinal functional brain development from birth, identifying early risk factors and assessing nutritional interventions (Jackson, Kennedy 2013). A recent piloted work among four to eight month old infants from the West Kiang District in Gambia, where agriculture is predominantly subsistence, replicated the patterns of selective cortical activation to adults performing social movements among UK infants of similar age (Lloyd-Fox et al. 2014). Importantly, fNIRS is relatively cheap and portable and therefore suitable for field-based neuroimaging research of cognitive development in infants and children in resource-poor rural settings (Lloyd-Fox et al. 2014).

\section{Box 13: Brain functional near infrared spectroscopy (fNIRS)}

Functional near infrared spectroscopy (fNIRS) is a non-invasive optical imaging technique which measures oxygenation changes in the brain resulting from neural activity in a response to external stimuli (Jackson, Kennedy 2013) such as visual, auditory, olfactory, and motor stimulation (Blasi et al. 2007). This indicates the activity level of different neural pathways and brain regions involved in basic as well as more complex cognitive functions (Blasi et al. 2007) such as object processing, social communication, human action and face processing (Lloyd-Fox et al. 2014). The technique is useful for studying longitudinal functional brain development from birth and developmental disorders (Lloyd-Fox et al. 2014). It is also a promising tool for identifying protective and risk factors for early childhood development (Lloyd-Fox et al. 2014) and for assessing nutritional interventions (Jackson, Kennedy 2013). fNIRS is non-invasive, affordable and portable (Blasi et al. 2007). 


\section{Early-life Stimulation}

The scientific community across multiple fields has acknowledged that direct care-givers within a family have the greatest influence on child cognitive and socio-emotional development (Heckman 2006). As already indicated, the family and the caregiving environment constitute a micro-system directly influencing child development via frequent interactions and activities. In the first years of life, the brain needs supporting and nurturing environments that give rise to high-quality positive experiences for optimal development to occur. When the developing brain is deprived of high quality stimulation, brain structure and function are compromised. In a high quality meta-analysis, Tanner et al. (2015) has indicated that early-stimulation interventions implemented in developing countries had the greatest causal and consistent positive effect over the spectrum of later-life cognitive outcomes (Tanner, Candland \& Odden 2015). To make the argument even stronger, Aboud and Yousafzai (2015) showed that psychosocial stimulation interventions had a medium effect on cognitive and language development compared to the relatively small effect of nutrition interventions on cognitive and language development; interventions aimed at improving situation at home have been especially successful, although group sessions, home and clinic visits have been beneficial as well (Aboud, Yousafzai 2015).

Based on the example of the Bucharest Early Intervention Project, institutionalized children who were randomized to be placed into foster care homes displayed gains in IQ scores at 8 years of age relative to children who remained institutionalized and experienced severe psychosocial deprivation. These effects were strongest for those children who remained with their intervention family and were present even among children who no longer stayed with the foster family at the time of assessment. Importantly, children assigned to the intervention group received greater stimulation than children who remained institutionalized. The foster parents were educated on early childhood development and encouraged to develop responsive care and high quality relationships with their child. See Box 14 for examples of possible interventions involving early stimulation.

\section{Box 14: Early-life Stimulation - Examples of Interventions:}

Example 1: IN KENYA, researchers are studying the impact of a program that distributes storybooks to poor households to see whether this helps improve children's readiness to succeed in primary school. Looking at pictures and reading helps stimulate visual and cognitive development.

http://www.worldbank.org/en/programs/sief-trust-fund/brief/kenya-emerge-reading

Example 2: IN BANGLADESH, researchers are studying the impact of a program that gives mothers and fathers information and encouragement on how to improve their children's development by playing, singing and talking to their babies and toddlers. Playing and singing to young children helps their brains develop and builds positive bonds between parents and their children.

http://www.worldbank.org/en/programs/sief-trust-fund/brief/building-parental-capacityto-help-child-nutrition-and-health

Example 3: IN COLOMBIA, a pilot program sought to improve children's brain development by showing caregivers how to stimulate the children using play and talk. The first follow-up survey in 2011 showed that the stimulation led to gains in children's cognitive development and in receptive language skills (which refers to the ability to understand and process what one hears or reads). Researchers are now doing a twoyear follow up to see if the gains have been sustained, which will help policymakers and development experts around the world improve the design of cost-effective and scalable strategies for successful early childhood development programs.

http://www.worldbank.org/en/programs/sief-trust-fund/brief/does-a-home-visitingprogram-in-early-childhood-have-sustained-effects-on-development-two-years-after-itends 


\section{POLICY INSTRUMENTS FROM A LIFE-COURSE PERSPECTIVE}

\section{Education}

Education is one of the established protective factors contributing to cognitive reserve (CR) across the life-course. The majority of studies conclude that education (mostly measured as years of education or educational attainment) provides reserve against age-associated brain pathology may delay the onset of dementia. However, the mechanisms of this relationship are less clear. Further research is required to examine under what conditions education is protective and what specific features of education, including the quality of education, might provide greater $\mathrm{CR}$.

While education is a powerful determinant of brain resilience across the life-course, substantial gender gaps in education remain globally as indicated in The World Bank's 2012 World Development Report on Gender Equality and Development (Wong 2012). Importantly, as highlighted in the very recent report titled "Women and Dementia - A global research review", dementia disproportionately affects women across the world (Erol, Brooker \& Peel 2015). More women than men develop the disease and are carers of individuals with dementia. In addition, nearly 60 percent of dementia cases live in low- and middle-income countries. Unsurprisingly then, gender disparities in educational opportunities might contribute to gender disparities in cognitive impairments and dementia outcomes.

For example, a recent study among elderly participants in central Nigeria reported that any primary education was protective against dementia and being a female was one of the major risks for developing dementia (Ochayi, Thacher 2006). Simultaneously, women in the study had lower educational attainment than men - this raises the possibility that gender differences in education partially explain the higher prevalence of dementia among women. At this time the evidence is only suggestive and requires substantial investigation. The notion of gender disparities within the concept of CR has not been sufficiently explored even in the countries such as the USA and UK where most of the relevant research takes place. To our knowledge, the concept of CR with a quantification of brain pathology has not been performed in any middle- or low-income country thus far; nor the issue of gender disparities. Ideally, such research would feature life-course determinants of $\mathrm{CR}$ including early childhood development factors. These efforts are especially important in the context of gender disparities in educational as well as occupational opportunities across the globe (Hausmann 2014).

\section{Box 15: Education - Examples of Interventions:}

Despite tremendous gains in access to education in recent years, millions of children still do not attend school and substantial gender gaps remain requiring immediate attention and action (King, 2011).

Example 1: Uganda is experiencing high population growth which means that an increasing number of children need to be placed in schools - currently 8 percent of children are out of school. The government of Uganda wants to ensure that all children attend school. However, the education sector is constrained by multiple challenges including a high level of teacher and student absenteeism, weak school level management structures, inadequate availability of learning materials, and large class sizes. The Uganda ministry of education is currently implementing a program funded by the Global Partnership for Education. As part of this program, the main goal is to strengthen the school system by increasing teachers and school effectiveness which in turn is hoped to improve learning outcomes in general.

http://www.globalpartnership.org/blog/uganda-wants-reach-all-out-school-children 
The development of job relevant skills, identified as the prerequisites for individual's occupational growth and growth of global economies at large, is a life-course journey. Importantly, the brain developed to its full potential is a prerequisite for the development of jobrelevant skills. The net effect of positive and negative early-life experiences on the developing brain and their accumulating impact over the life-course shapes the development of complex cognitive and socio-emotional functions. Strong cognitive and socio-emotional functioning are the prerequisite for acquisition and development of job-relevant skills (Banerji et al. 2010) and thus entry into better jobs and healthier environments.

In the context of cognitive reserve (CR), some of the life-course determinants of job-relevant skills provide our brains with resilience to adversities. These are: greater childhood cognitive ability (shaped by early childhood environment), higher education, more complex occupations (Staff et al. 2004b) relying on technological advancements, and resilient personalities. Also within this framework, keeping individuals in quality occupations for longer is not only beneficial for economic growth but for healthy brain aging. Occupations which challenge the brain in later-life maintain structure and function of the brain. Overall, the "STEP Skills Measurement" framework (Banerji et al. 2010) reflects features of the developing brain and its importance in job-relevant skills formation (see Figure 5).

Figure 5: Many of the life-course determinants of cognitive reserve (CR) are mapped onto to the "STEP Skills Measurement" framework (Banerji et al. 2010).

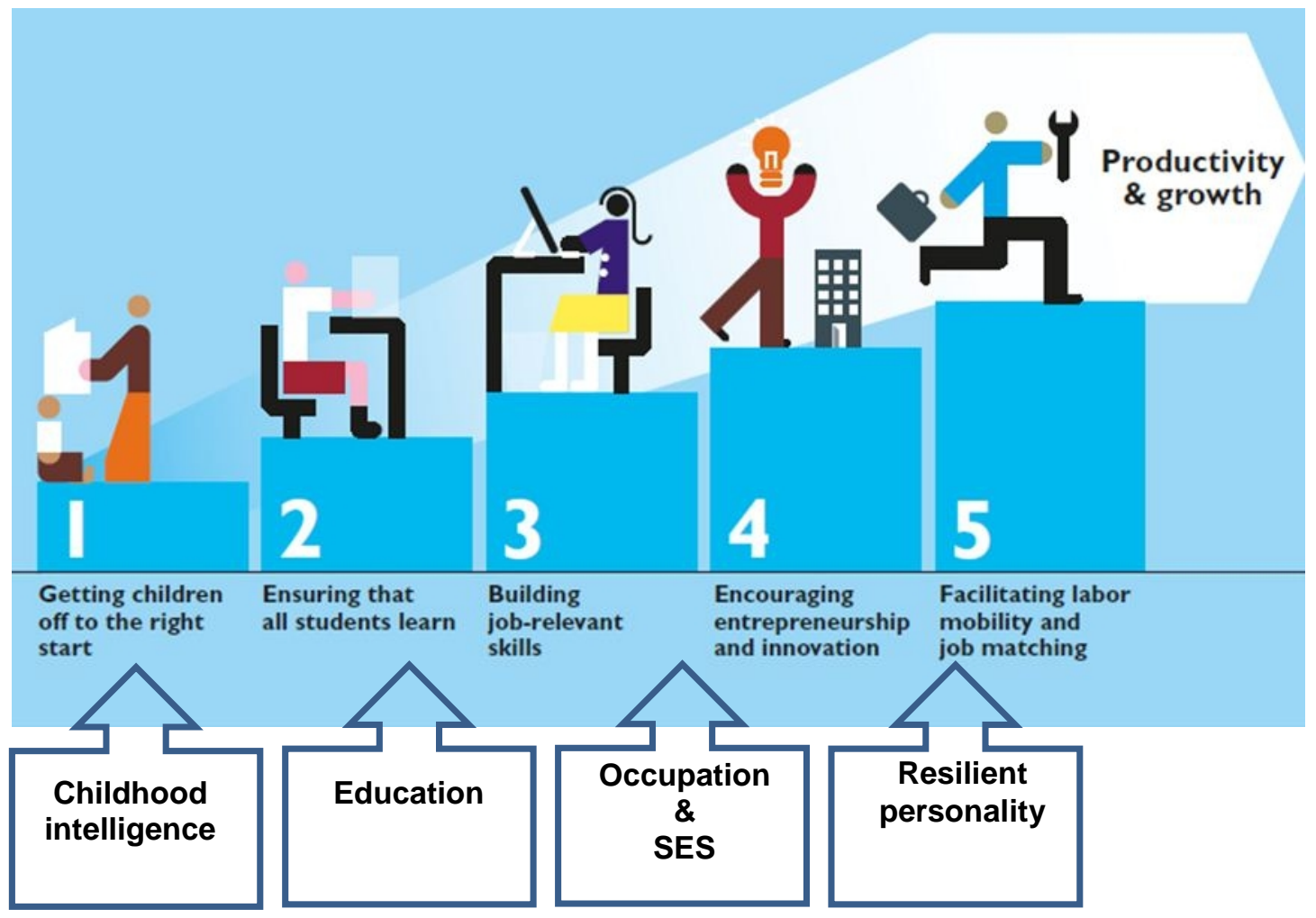

Source: The main figure in (Banerji et al. 2010). The bottom part of the figure: Author. 
Job-relevant skills determine the nature of employment which in turn impact brain structure and function in later-life. Occupational characteristics mitigate against the negative influences of neuropathology in non-demented (Staff et al. 2004a) as well as demented populations (Garibotto et al. 2008). Multiple studies suggest beneficial effects of occupational activities on cognitive performance in advanced age. For example, (Adam et al. 2013) showed that professionally active individuals scored much higher on cognitive tests than their never-employed or retired counterparts. Also, greater occupational complexity (Andel et al. 2007) and more job control (Andel et al. 2011) predicted higher summary cognitive scores. Furthermore, lower odds for developing cognitive impairment are associated with superior occupational positions within a social class hierarchy (LI, WU \& SUNG 2002) as well as more complex (Andel et al. 2007), more mentally demanding (Dartigues et al. 1992), and more active jobs characterized by high control/high demands (Andel et al. 2011).

Box 16: Job Stimulating Environment - Examples of Interventions:

\section{Example 1: Job re-design as a potential approach to address socio-economic disparities in health and brain outcomes.}

In late 90s, Volvo replaced a more traditional assembly line with a more flexible work organization (Melin, Lundberg, Söderlund, \& Granqvist, 1999). The former one was characterized by fixed work stations organized as a chain and involved short repetitive work cycles; the latter one included small autonomous groups whose members had the opportunity to influence the pace and work activities. By introducing the more flexible work organization, the aim was to increase the amount of control and social interactions between workers, to decrease the monotony of the traditional assembly line work and to keep the production level constant.

Workers assigned to the more flexible group reported significantly more variation, independence and abilities to learn new skills at work. Workers at the traditional assembly line had significantly increased various stress indicators including systolic blood pressure, heart rate and the levels of adrenaline ("stress hormone") during the work shifts. In summary, activities performed at the flexible work organization were less stressful and overall more beneficial, especially among female workers. Although the direct effects of this intervention on the brain were not measured, beneficial effects on brain functions and structure are plausible.

Example 2: In the Dominican Republic, the government is offering classroom-based vocational and life skills training, combined with on-the-job internships, for at-risk youth ages 16-29 living in the poorest areas. Results from a rigorous impact evaluation showed that the graduates had higher chances of acquiring a formal job and a larger income, particularly among women. At 12-18 months after completion of the program, female participants were more likely to be employed, earn more and find satisfaction in their work compared to those in a control group.

http://www.worldbank.org/en/results/2014/04/08/more-promising-future-poor-youthdominican-republic http://www.worldbank.org/en/topic/socialprotectionlabor/brief/skills-for-jobs 


\section{CONCLUSIONS AND POLICY IMPLICATIONS}

"Human development" and "development" are inseparable, and now new evidence emerges that "brain development" is the key driving mechanism behind this association. As many low- and middle-income countries are entering the trajectory of economic growth, early childhood interventions should move away from the philosophy of "survival" to "thriving" and recognize the importance of the developing brain. The brain, the source of our cognition and emotions, is arguably the most essential organ in the human body and the key feature of early childhood development. Brain complexity programmed in a person's early-life gives rise to cognitive and socio-emotional functions which fuse together to form skills acquired across the life-course. Achieving the brain's full developmental potential is crucial for a lifetime of individual and societal well-being and success.

Early childhood development does not occur within a vacuum; this complex process is influenced by the interplay between individual characteristics, biological forces, family and class dynamics imbedded within broader historical, socio-cultural and economic circumstances and other environmental factors. Positive and negative experiences in early-life affect childhood development through changes in brain structure and function. The experiences affecting function and structure of the brain across the life-course emerge from a social world - therefore many of the environmental factors affecting the brain are potentially modifiable and could be a target for interventions. The brain needs supporting and nurturing environments that give rise to positive experiences for optimal development to occur. A healthy and active brain, shaped by adequate nutrition and safe and enriching environments in early-life, enables retention of brain functioning across the lifespan and healthy brain aging.

Adverse environments and socio-psychological hardship associated with poverty or disadvantaged socio-economic circumstances are the source of stress having a detrimental impact on a child's developing brain. Understanding the neural mechanisms of early-life socioeconomic disparities in brain function is crucial for the design of effective and scalable early interventions. However, this research is just in its infancy. Early-life socio-economic disparities affect multiple brain functions that are important for school readiness skills including language, working memory, cognitive control (executive functions), mental flexibility, and self-regulation. The resulting socioeconomic differences in neural development and brain functioning begin to emerge already in infancy; if no intervention is provided at the right point in time, they persist and even widen with increasing age. Although mechanisms of brain recovery upon early interventions are complex, there is "clear evidence" that protective early-life factors (or removal of risk factors) can positively impact the brain and ameliorate adverse outcomes (Bick et al. 2015).

On the positive side, the brain is capable of developing resilience mechanisms even to severe early-life neglect if provided with the opportunity to continue development in caring and enriching environments. Generally, because of the brain's decreasing malleability across the life-course, the earlier sustained positive experiences impact the brain, the better for the brain. The associations between socio-economic circumstances and brain structure and function could emerge from the differences in prenatal environment (maternal health) as well as postnatal experiences or exposures: (family) stress, cognitive stimulation, and nutrition. All of these factors could constitute potential targets for interventions.

Development and aging are part of a continuum. An increasing number of countries face the challenge of aging societies associated with the burden of impairments on individuals, health care providers, society and the states. The developing countries are being affected the most. Identifying vulnerable groups, potential interventions and the "windows of opportunities" when these are best applied are becoming urgent priorities as populations across the globe age. Recent high quality studies involving brain imaging have shown that those from the most disadvantaged backgrounds, who will have potentially greatest brain developmental delays and greater brain pathology in latelife, need the early-life investment most. Special attention and early-life investment should be also directed at children growing up in institutional settings. 
The maximum brain development should be sustained with life-course investments and equal opportunities for highest returns. Many of the qualities of brain resilience to biological and social factors are shaped in early life when the brain is most plastic. However, the window of opportunity is not confined to early childhood. Brain development is cumulative and continues through childhood and adolescence. These changes are as important as the ones that occur before age 3 offering multiple opportunities for interventions in later stages of development when more complex cognitive and socio-emotional functions are shaped. With the appropriate investments extending beyond early-life resilience can be sustained across the lifespan. The matter is not only biological but also social in nature - life-course factors such as early-life cognitive ability (which determines maximum life-time cognition), greater educational attainment, job-relevant skills and more complex occupations provide individuals with resilience to brain declines associated with aging. Brain development across the life-course calls for neuroscience-informed interventions to increase the chances for brain's recovery and boost the brain's resilience to future insults. High quality impact evaluations of such interventions are needed to determine what interventions work best, when and in what context.

\section{Box 17: Key messages:}

1) Positive and negative experiences in early-life affect childhood development through changes in brain structure and function. A healthy and active brain, shaped by adequate nutrition and safe and enriching environments starting in utero, enables retention of brain functioning across the lifespan and healthy brain aging.

2) Brain complexity programmed in a person's early-life gives rise to cognitive and socio-emotional functions which fuse together to form skills acquired across the life-course. Cognitive and socio-emotional functions complement each other; it is thus important to ensure that interventions targeting human development and skills acquisition are holistic and target both cognitive and socio-emotional domains.

3) Brain systems affected by early-life socioeconomic adversities play a critical role in domains important for children's school readiness skills. The resulting socioeconomic differences in neural development and brain functioning begin to emerge already in infancy; if no intervention is provided at the right point in time, they persist and even widen with increasing age. Generally, because of the brain's decreasing malleability across the life-course, the earlier sustained and high quality experiences impact the brain, the better for the brain.

4) Many of the qualities of brain resilience to biological and social factors are shaped in early life when the brain is most plastic. With the appropriate investments extending beyond early-life for example, in quality education and jobs, resilience can be sustained across the life-course. Therefore the maximum brain development should be sustained with life-course investments and equal opportunities for highest returns.

5) Everyone deserves a good head-start and the attainment of optimal brain development to become resilient to potential mid-life adversities and aging process. Individuals from the most disadvantaged backgrounds, who will have greatest developmental delays and brain burden, need the early-life investment the most.

6) Because children in developing countries are exposed to multiple risks to brain development at once, it is very unlikely that a single-factor intervention will solve the issue of suboptimal brain development with its life-course consequences; rather, multi-factor interventions incorporating the features of behavioral change might produce the expected results (Aboud \& Yousafzai, 2015). 
It is important to ensure that interventions targeting skills acquisition and development across the lifespan are holistic and target both cognitive and socio-emotional domains. Some components of brain function remain resilient to aging processes or even improve with time but the other have a tendency to decline. While it makes sense to recognize these brain shifts in order to design appropriate labor market strategies among currently aging populations across the world, let's not rely only on skills that remain intact or even improve with time. Not only due to the complementary nature of cognitive and socio-emotional skills but also because the fluid aspects of cognition are sensitive to aging processes. There is evidence that complex brain tasks such as social interactions (Ybarra et al. 2008) have a positive effect on cognitive aging. Also, interventions directed at cognitive domains may change personality traits such as openness to experience (Jackson et al. 2012) leading to an active and engaged lifestyle (Hogan et al. 2012). Keeping these findings in mind, how about designing an intervention directed specifically at job skills acquisition? Such holistic approach might bring not only job market returns but also savings in other sectors such as healthcare due to high costs associated with dementia management.

As the costs of neuroimaging falls, future rigorous evaluations of interventions targeting skills development could incorporate measurement of brain functioning and structure. Imaging techniques are useful for studying longitudinal structural and functional brain development from birth and for developmental disorders (Lloyd-Fox et al. 2014). They are also promising tools for identifying protective and risk factors for early childhood development (Lloyd-Fox et al. 2014). Some of the new technologies are relatively cheap and portable and therefore suitable for fieldbased neuroimaging research of brain development in infants and children in resource-poor rural settings (Lloyd-Fox et al. 2014). Importantly, existing behavioral paradigms and the wellestablished assessment tools designed to measure cognitive and socio-emotional functioning can only detect the impact of environmental factors once they reach the threshold for observable deficits (Lloyd-Fox et al. 2014). Imaging techniques are more culturally-neutral markers for brain outcomes and their adaptation as well as standardization are more straight-forward than for the more conventional tools, therefore may play a pivotal role in informing targeted early intervention strategies (Lloyd-Fox et al. 2014).

With the rapid advancement of current technologies, the promising call for utilizing brain biomarkers for the assessment of ECD educational and nutritional interventions is very recent and awaits further actions (Pavlakis et al. 2015) (Noble, Farah 2013) (Lloyd-Fox et al. 2014). Performing neuroimaging (or electrophysiology) in parallel to ECD cognitive and socioemotional tests would allow us to better understand interventions (e.g. educational programs) and their efficacy in children with the potentially sustained effects across the life-course (Pavlakis et al. 2015) (Lloyd-Fox et al. 2014). Because some neurobiologic correlates of socioeconomic disparities are already known, imaging and/or electrophysiology methods could be applied after implementation of a specific early intervention designed to reduce SES disparities.

While our interdisciplinary knowledge in this area has grown substantially in recent years, important gaps remain. Much of what we know about brain's resilience is based on participants from high income countries. We know very little about the effects of poverty on the developing brain structure and function in low-resource settings, although some exciting initiatives are starting. Even less is known about brain development in fragile states and conflict areas. It is challenging to identify scalable low-cost approaches in culturally diverse context to eliminate the disparities in brain development. These efforts demand out-of-the-box solutions and will require collaboration across multiple disciplines and sectors. Also, there are virtually no randomized control design studies performed within the context of cognitive reserve involving quantification of brain burden. Most of the associations are correlational while there are opportunities to accommodate interventions as part of the high quality UK population cohort data ${ }^{1}$ such as Aberdeen Children of the $1950 \mathrm{~s}^{2}$. Evaluations of interventions targeting cognitive and socio-emotional skills development

${ }^{1}$ http://www.mrc.ac.uk/research/facilities/cohort-directory/

${ }^{2}$ http://www.abdn.ac.uk/birth-cohorts/1950s/ 
might be a possibility. Are grit and psycho-social resilience only relevant when some base for cognitive and socio-emotional abilities is achieved? Are the reserve processes at work when the brain is exposed to extreme forms of poverty? Does early-life resilience to adversities come at a cost in later life (Kuh et al. 2003)? These answers can greatly enhance our ability to carry out costeffective policies to eliminate extreme poverty and improve shared prosperity. Everyone deserves a good head-start and the attainment of optimal brain development to become resilient to potential mid-life adversities and aging process.

\section{Box 18: Future perspectives:}

1) Much of what we know about brain's resilience is based on participants from high income countries. We know very little about the effects of poverty on the developing brain structure and function in low-resource settings. Even less is known about brain development in fragile states and conflict areas. These questions are important to identify interventions which are feasible in lowresource and conflict settings.

2) It is challenging to identify scalable low-cost approaches in culturally diverse context to eliminate the disparities in brain development. These efforts demand out-of-the-box solutions and will require collaboration across multiple disciplines and sectors.

3) As the costs of neuroimaging and other modalities falls, future rigorous quality assessment and evaluations of interventions targeting skills development could incorporate measurement of brain functioning and structure. Performing neuroimaging (or electrophysiology) in parallel to cognitive and socio-emotional tests would allow us to better understand interventions (e.g. educational programs) and their efficacy in children (and the potentially sustained effects across the life-course). The call for utilizing brain biomarkers for the assessment of ECD interventions is very recent (Pavlakis et al. 2015, Noble and Farah 2013) and awaits further actions. These answers can greatly enhance our ability to carry out cost-effective policies to eliminate extreme poverty and improve shared prosperity.

4) The life-course theory acknowledges biological and socio-cultural turning points in life as windows of opportunity for interventions. The window of opportunity is not confined to early childhood. The brain, although to a lesser extent, remains malleable across the life-course. More evidence from brain sciences is needed to determine specific timing of interventions to increase the chances for brain's recovery and/or boost the brain's resilience to future insults.

5) Most of the associations are correlational while there are opportunities to accommodate interventions as part of the already existing high quality population cohorts. Evaluations of interventions targeting cognitive and socioemotional skills development might be a possibility. 


\section{REFERENCES}

Aboud, F. E. and A, K, Yousafzai. 2015. "Global Health and Development in Early Childhood." Annual Review of Psychology, vol. 66, pp. 433-457.

Adam, S., E. Bonsang, C. Grotz, and S. Perelman. 2013. "Occupational Activity and Cognitive Reserve: Implications in Terms of Prevention of Cognitive Aging and Alzheimer's Disease." Clinical Interventions in Aging. vol. 8, pp. 377-390.

Andel, R., M. Crowe, I. Kareholt, J. Wastesson and M. G. Parker. 2011. "Indicators of Job Strain at Midlife and Cognitive Functioning in Advanced Old Age." The Journals of Gerontology. Series B, Psychological Sciences and Social Sciences. vol. 66, no. 3, pp. 287-291.

Andel, R., I. Kareholt, M. G Parker, M. Thorslund and M. Gatz. 2007. "Complexity of Primary Lifetime Occupation and Cognition in Advanced Old Age." Journal of Aging and Health, vol. 19, no. 3, pp. 397-415.

Banerji, A., W. Cunningham, A. Fiszbein, E. King, H. Patrinos, D. Robalino, and J. Tan. 2010. Stepping Up Skills: For More Jobs and Higher Productivity, World Bank.

Bartley, A.J., D. W. Jones and D. R. Weinberger. 1997. "Genetic Variability of Human Brain Size and Cortical Gyral Patterns." Brain: A Journal of Neurology, vol. 120 ( Pt 2), no. Pt 2, pp. 257-269.

Bick, J. and C. A. Nelson. 2016. "Early Adverse Experiences and the Developing Brain." Neuropsychopharmacology: Official Publication of the American College of Neuropsychopharmacology, vol. 41, no. 1, pp. 177-196.

Bick, J., T. Zhu, C. Stamoulis, N. A. Fox, C. Zeanah and C. A. Nelson. 2015. "Effect of Early Institutionalization and Foster Care on Long-term White Matter Development: A Randomized Clinical Trial." JAMA Pediatrics, vol. 169, no. 3, pp. 211-219.

Boyden, J., S. Dercon and A. Singh. 2014. "Child Development in a Changing World: Risks and Opportunities." The World Bank Research Observer.

Brito, N. H and K. G. Noble. 2014, "Socioeconomic Status and Structural Brain Development." Frontiers in Neuroscience, vol. 8, pp. 276.

Camerer, C., G. Loewenstein and D. Prelec. 2005. "Neuroeconomics: How Neuroscience can Inform Economics." Journal of Economic Literature, pp. 9-64.

Chemin, M., J. De Laat and J. Haushofer. 2013. "Negative Rainfall Shocks Increase Levels of the Stress Hormone Cortisol Among Poor Farmers in Kenya." Available at SSRN 2294171.

Dartigues, J., M. Gagnon, L. Letenneur, P. Barberger-Gateau, D. Commenges, M. Evaldre and R. Salamon. 1992. "Principal Lifetime Occupation and Cognitive Impairment in a French Elderly Cohort (Paquid)." American Journal of Epidemiology, vol. 135, no. 9, pp. 981-988.

Deary, I. J., J. Corley, A. J. Gow, S. E. Harris, L. M. Houlihan, R. E. Marioni, L. Penke, S. B. Rafnsson and J. M. Starr. 2009. "Age-associated Cognitive Decline." British Medical Bulletin, vol. 92, pp. 135-152.

Deary, I. J., L. Penke, and W. Johnson. 2010. "The Neuroscience of Human Intelligence Differences." Nature Reviews. Neuroscience, vol. 11, no. 3, pp. 201-211. 
Double, K. L., S. Reyes, E. L. Werry and G. M. Halliday. 2010. "Selective Cell Death in Neurodegeneration: Why are Some Neurons Spared in Vulnerable Regions?"

Progress in Neurobiology, vol. 92, no. 3, pp. 316-329.

Duckworth, A. L., C. Peterson, M. D. Matthews, and D. R. Kelly. 2007. "Grit:

Perseverance and Passion for Long-term Goals." Journal of Personality and Social Psychology, vol. 92, no. 6, pp. 1087-1101.

Erol, R., D. Brookerand E. Peel. 2015. "Women and Dementia: A Global Research Review."

Farmer, P. E., B. Nizeye, S. Stulacand S. Keshavjee. 2006. "Structural Violence and Clinical Medicine." PLoS Medicine, vol. 3, no. 10, pp. e449.

Fernald, A., V. A. Marchman, and A. Weisleder. 2013. "SES S

Differences in Language Processing Skill and Vocabulary are Evident at 18 Months." Developmental Science. vol. 16, no. 2, pp. 234-248.

Fernald, L.C., A. Weber, E. Galasso and L. Ratsifandrihamanana. 2011. "Socioeconomic Gradients and Child Development in a Very Low Income Population: Evidence from Madagascar." Developmental Science, vol. 14, no. 4, pp. 832-847.

Garibotto, V., B. Borroni, E. Kalbe, K. Herholz, E. Salmon, V. Holtoff S. Sorbi, S. F. Cappa, A. Padovani, F. Fazio, Perani, D. 2008. "Education and Occupation as Proxies for Reserve in a MCI Converters and AD: FDG-PET Evidence." Neurology, vol. 71, no. 17, pp. 1342-1349.

Gazzaniga, M. S., K. W. Doron and C. M. Funk. 2009. "Looking Toward the Future: Perspectives on Examining the Architecture and Function of the Human Brain as a Complex System." in The Cognitive Neurosciences, ed. M.S. Gazzaniga, 4th ed. edn, MIT Press, Cambridge, Mass., pp. 1247-1254.

Gazzaniga, M. S., R. B. Ivry G. R. Mangun. 2009. Cognitive Neuroscience: The Bbiology of the Mind, 3rd edn, W.W. Norton \& Co., New York.

Gianaros, P. J., J. A. Horenstein, A. R. Hariri, L. K. Sheu, S. B. Manuck, , K. A. Matthews and S. Cohen. 2008. "Potential Neural Embedding of Parental Social Standing." Social Cognitive and affective Neuroscience, vol. 3, no. 2, pp. 91-96.

Gianaros, P. J. and S. B. Manuck. 2010. "Neurobiological Pathways Linking Socioeconomic Position and Health." Psychosomatic Medicine, vol. 72, no. 5, pp. 450-461.

Grantham-McGregor, S., Y. B. Cheung, S. Cueto, P. Glewwe, L. Richter, B. Strupp and International Child Development Steering Group. 2007. "Developmental Potential in the First 5 Years for Children in Developing Countries." The lancet, vol. 369, no. 9555, pp. 60-70.

Hair, N. L., J. L. Hanson, B. L. Wolfe and S. D. Pollak. 2015. "Association of Child Poverty, Brain Development, and Academic Achievement", JAMA Pediatrics, vol. 169, no. 9, pp. 822-829.

Hausmann, R. 2014. "Gender Gap Report 2014."

Heckman, J. J. 2006. "Skill Formation and the Economics of Investing in Disadvantaged cCildren." Science (New York, N.Y.), vol. 312, no. 5782, pp. 1900-1902.

Hogan, M. J., R. T. Staff, B. P. Bunting, I. J. Deary and L. J. Whalley. 2012. "Openness to Experience and Activity Engagement Facilitate the Maintenance of Verbal Ability in Older Adults." Psychology and Aging, vol. 27, no. 4, pp. 849-854. 
Ivanovic, D. M., B. P. Leiva, H. T. Perez, Inzunza, N. B., A. F. Almagia , T. D. Toro, M. S. Urrutia, J. O. Cervilla and E. O. Bosch. 2000. "Long-term Effects of Severe Undernutrition During the First Year of Life on Brain Development and Learning in Chilean High-school Graduates." Nutrition (Burbank, Los Angeles County, Calif.), vol. 16, no. 11-12, pp. 1056-1063.

Jackson, J. J., P. L. Hill, B. R. Payne, B. W. Roberts and E. A. Stine-Morrow. 2012. "Can an Old Dog Learn (and want to experience) New Tricks? Cognitive Training Increases Openness to Experience in Older Adults." Psychology and Aging, vol. 27, no. 2, pp. 286-292.

Jackson, P.A D. O. Kennedy. 2013. "The Application of Near Infrared Spectroscopy in Nutritional Intervention Studies." Frontiers in Human Neuroscience, vol. 7, pp. 473.

Kim, P., G. W. Evans, M. Angstadt, S. S. Ho, C. S. Sripada, J. E. Swain, I. Liberzonand K. L. Phan. 2013. "Effects of Childhood Poverty and Chronic Stress on Emotion Regulatory Brain Function in Adulthood." Proceedings of the National Academy of Sciences of the United States of America, vol. 110, no. 46, pp. 18442-18447.

Kolb, B., and R. Gibb. 2011, "Brain Plasticity and Behaviour in the Developing Brain." Journal of the Canadian Academy of Child and Adolescent Psychiatry. Journal de l'Academie Canadienne de Psychiatrie de L'enfant et de L'adolescent, vol. 20, no. 4, pp. 265-276.

Kuh, D., Y. Ben-Shlomo, J. Lynch, J. Hallqvist and C. Power. 2003. "Life Course Epidemiology." Journal of Epidemiology and Community Health. vol. 57, no. 10, pp. 778-783.

Levitt, P. 2003. "Structural and Functional Maturation of the Developing Primate Brain." The Journal of Pediatrics, vol. 143, no. 4 Suppl, pp. S35-45.

Li, C., S. C. Wu, and F. Sung. 2002. "Lifetime Principal Occupation and Risk of Cognitive Impairment among the Elderly." Industrial Health, vol. 40, no. 1, pp. 713.

Lloyd-Fox, S., M. Papademetriou, M. K. Darboe, N. L. Everdell, R. Wegmuller, A. M. Prentice, S. E. Moore and C. E. Elwell. 2014. "Functional Near Infrared Spectroscopy (fNIRS) to Assess Ccognitive Function in Infants in Rural Africa." Scientific Reports, vol. 4, pp. 4740.

Luby, J. L. 2015. "Poverty's Most Insidious Damage: The Developing Brain", JAMA Pediatrics, vol. 169, no. 9, pp. 810-811.

Maercker, A. and S. Forstmeier. 2012. Healthy Brain Ageing: The New Concept of Motivational Reserve.

Maguire, E.A., D. G. Gadian, I. S. Johnsrude, C. D. Good, J. Ashburner , R. S. Frackowiak and C. D. Frith, C.D. 2000. "Navigation-related Structural Change in the Hippocampi of Taxi Drivers." Proceedings of the National Academy of Sciences of the United States of America, vol. 97, no. 8, pp. 4398-4403.

McEwen, B.S., and P. J. Gianaros. 2010. "Central Role of the Brain in Stress and Adaptation: Links to Socioeconomic Status, Health, and Disease." Annals of the New York Academy of Sciences, vol. 1186, pp. 190-222.

Miller, E. K., and J. D. Cohen. 2001. "An Integrative Theory of Prefrontal Cortex Function." Annual Review of Neuroscience, vol. 24, pp. 167-202.

Mullainathan, S., and E. Shafir. 2014. "Freeing up Intelligence." Scientific American Mind, vol. 25, no. 1, pp. 58-63. 
Murray, A.D. 2012, "Imaging Approaches for Dementia." AJNR. American Journal of Neuroradiology, vol. 33, no. 10, pp. 1836-1844.

Murray, A. D., C. J. McNeil, S. Salarirad, L. J. Whalleyand R. T. Staff. 2014, "Early Life Socioeconomic Circumstance and Late Life Brain Hyperintensities--a Ppopulation based Cohort Study." PloS one, vol. 9, no. 2, pp. e88969.

Murray, A.D., R. T. Staff, C. J. McNeil., S. Salarirad, T. S. Ahearn, N. Mustafa and L. J. Whalley. 2011. "The Balance Between Cognitive Reserve and Brain Imaging Biomarkers of Cerebrovascular and Alzheimer's Diseases." Brain: A Journal of Neurology, vol. 134, no. Pt 12, pp. 3687-3696.

Murray, A.D., E. T. Staff, S. D. Shenkin, I. J. Deary, J. M. Starr andL. J. Whalley. 2005. "Brain White Matter Hyperintensities: Relative Importance of Vascular Risk Factors in Nondemented Elderly People." Radiology, vol. 237, no. 1, pp. 251-257.

Mustafa, N., T. S. Ahearn, G. D. Waiter, A. D. Murray, L. J. Whalley, and R. T. Staff. 2012. "Brain Structural Complexity and Life Course Cognitive Change." NeuroImage, vol. 61, no. 3, pp. 694-701.

National, S. C. 2014. "Excessive Stress Disrupts the Development of Brain Aarchitecturenull." Journal of Children's Services, vol. 9, no. 2, pp. 143-153.

Naudeau, S., N. Kataoka, A. Valerio, M. J. Neuman andL. K. Elder. 2011. "Investing in Young Children: An Early Childhood Development Guide for Policy Dialogue and Project Preparation."

Nelson, C.A., C. H. Zeanah, N. A. Fox, P. J. Marshall, A. T. Smyke andD. Guthrie. 2007. "Cognitive Recovery in Socially Deprived Young Children: The Bucharest Early Intervention Project." Science (New York, N.Y.), vol. 318, no. 5858, pp. 19371940.

Nelson, C.A. 1999. "How Important are the First 3 Years of Life?", Applied Developmental Science, vol. 3, no. 4, pp. 235-238.

Nelson, C.A., N. A. Fox., C. H. Zeanah I. Ebrary. I. 2014. Romania's Abandoned Children: Deprivation, Brain Development, and the Struggle for Recovery. Harvard University Press, Cambridge, Massachusetts ; London, England.

Nisbett, R. E., J. Aronson, C. Blair, W. Dickens, Flynn, J., D. F. Halpern. and E. Turkheimer. 2012. "Intelligence: New Findings and Theoretical Developments." The American Psychologist, vol. 67, no. 2, pp. 130-159.

Noble, K.G. 2014. "Rich Man, Poor Man: Socioeconomic adversity and brain development", Cerebrum: The Dana Forum on Brain Science, vol. 2014, pp. 6-Jun.

Noble, K.G., Engelhardt, L. E., Brito, N. H., Mack, L. J., Nail, E.J., Angal, J., Barr, R., Fifer, W. P., Elliott, A. J. and PASS Network. 2015. "Socioeconomic Disparities in Neurocognitive Development in the First Two Years of Life." Developmental Psychobiology, vol. 57, no. 5, pp. 535-551.

Noble, K. G. and M. J. Farah. 2013. "Neurocognitive Consequences of Socioeconomic Disparities: The Intersection of Cognitive Neuroscience and Public Health." Developmental Science, vol. 16, no. 5, pp. 639-640.

Noble, K. G., S. M. Houston, N. H. Brito, H. Bartsch, E. Kan, J. M. Kuperman, N. Akshoomoff, D. G. Amaral, C. S. Bloss, O. Libiger, N. J. Schork, S. S. Murray, B. J. Casey, L. Chang, T. M. Ernst, J. A. Frazier, J. R. Gruen, D. N. Kennedy, P. Van Zijl, S. Mostofsky, W. E. Kaufmann, T. Kenet, A. M. Dale, T. L. Jernigan, and E. R. 
Sowell. 2015. "Family Income, Parental Education and Brain Structure in Children and Adolescents." Nature Neuroscience, vol. 18, no. 5, pp. 773-778.

Noble, K. G., B. D. McCandliss, M. J. Farah. 2007. "Socioeconomic Gradients Predict Individual Differences in Neurocognitive Abilities", Developmental Science, vol. 10, no. 4, pp. 464-480.

Ochayi, B. and T. D. Thacher. 2006. "Risk Factors for Dementia in Central Nigeria." Aging and Mental Health, vol. 10, no. 6, pp. 616-620.

Okon-Singer, H., T. Hendler, L. Pessoa, A. J. Shackman. 2015. "The Neurobiology of Emotion-cognition Interactions: Fundamental Questions and Strategies for Future Research." Frontiers in Human Neuroscience. vol. 9, pp. 58.

Pavlakis, A. E., K. Noble, S. G. Pavlakis, N. Ali and Y. Frank. 2015, "Brain Imaging and Electrophysiology Biomarkers: Is there a Role in Poverty and Education Outcome Research?" Pediatric Neurology, vol. 52, no. 4, pp. 383-388.

Pessoa, L. 2008. "On the Relationship between Emotion and Cognition." Nature Reviews. Neuroscience, vol. 9, no. 2, pp. 148-158.

Petitto, L. 2005. "How the Brain Begets Language." The Cambridge Companion to Chomsky, pp. 84-101.

Phelps, Elizabeth A., and R. Delgado, Mauricio. 2009. "Emotion and Decision Making" in The Cognitive Neurosciences, ed. M.S. Gazzaniga, 4th ed. edn, MIT Press, Cambridge, Mass., pp. 1093-1103.

Rakic P., J. I. Arellano, J. J. Breunig. 2009. "Development of the Primate Cerebral Cortex." in The Cognitive Neurosciences, ed. Michael S Gazzaniga, 4th ed. edn, MIT Press, Cambridge, Mass., pp. 7-28.

Richards, M. and S. L. Hatch. 2011. "A Life Course Approach to the Development of Mental Skills." The Journals of Gerontology. Series B, Psychological Sciences and Social Sciences, vol. 66 Suppl 1, pp. i26-35.

Sheridan, M.A., N. A. Fox, C. H. Zeanah, K. A. McLaughlin and C. A. Nelson. 2012. "Variation in Neural Development as a Result of Exposure to Institutionalization Early in Childhood." Proceedings of the National Academy of Sciences of the United States of America, vol. 109, no. 32, pp. 12927-12932.

Shonkoff, J. P., A. S. Garner, Committee on Psychosocial Aspects of Child and Family Health, Committee on Early Childhood, Adoption, and Dependent Care and Section on Developmental and Behavioral Pediatrics. 2012. "The Lifelong Effects of eErly Childhood Adversity and Toxic Stress." Pediatrics, vol. 129, no. 1, pp. e232-46.

Silvia A. Bunge., P. Allyson Mackey and J. Kirstie Whitaker. 2009. "Brain Changes Underlying the Development of Cognitive Control and Reasoning." in The Cognitive Neurosciences, ed. M.S. Gazzaniga, 4th ed. edn, MIT Press, Cambridge, Mass., pp. 73-85.

Small, D.H., S. S. Mok and J. C. Bornstein. 2001. "Alzheimer's Disease and Abeta Toxicity: From top to Bottom." Nature Reviews. Neuroscience, vol. 2, no. 8, pp. 595-598.

Staff, R.T., A. D. Murray, T. S. Ahearn, N. Mustafa, H. C. Fox and L. J. Whalley. 2012. "Childhood Socioeconomic Status and Adult Brain Size: Childhood Socioeconomic Status Influences Adult Hippocampal Size." Annals of Neurology, vol. 71, no. 5, pp. 653-660. 
Staff, R.T., A. D. Murray, I. J. Dearyand L. J. Whalley. 2004a. "What Provides Cerebral Reserve?", Brain: A Journal of Neurology, vol. 127, no. 5, pp. 1191-1199. . 2004b. "What Provides Cerebral Reserve?" Brain: A Journal of Neurology, vol. 127, no. Pt 5, pp. 1191-1199.

Stern, Y. 2012. "Cognitive Reserve in Ageing and Alzheimer's Disease." The Lancet. Neurology, vol. 11, no. 11, pp. 1006-1012.

Thompson, R.A., and C. A. Nelson. 2001. "Developmental Science and the Media. Early Brain Development." The American Psychologist, vol. 56, no. 1, pp. 5-15.

Tomalski, P., and M. H. Johnson. 2010. "The Effects of Early Adversity on the Adult and Developing Brain." Current Opinion in Psychiatry, vol. 23, no. 3, pp. 233-238.

Tomalski, P., D. G. Moore, H. Ribeiro, E. L. Axelsson, E. Murphy, A. Karmiloff-Smith, M. H. Johnson and E. Kushnerenko. 2013. "Socioeconomic Status and Functional Brain Development - Associations in Early Infancy." Developmental Science, vol. 16, no. 5, pp. 676-687.

Van den Bergh, B.R. 2011, "Developmental Programming of Early Brain and Behaviour Development and Mental Health: A Conceptual Framework." Developmental Medicine and Child Neurology, vol. 53 Suppl 4, pp. 19-23.

Victora, C. G., M. de Onis, P. C. Hallal, M. Blossnerand R. Shrimpton. 2010. "Worldwide Timing of Growth Faltering: Revisiting Implications for Interventions." Pediatrics, vol. 125, no. 3, pp. e473-80.

Waber, D. P., C. P. Bryce, J. M. Girard, M. Zichlin, G. M. Fitzmaurice, and J. R. Galler. 2014. "Impaired IQ and Academic Skills in Adults who Experienced Moderate to Severe Infantile Malnutrition: A 40-year Study." Nutritional Neuroscience, vol. 17, no. 2, pp. 58-64.

Walker, S. P., T. D. Wachs, J. M. Gardner, B. Lozoff, G. A. Wasserman,E. Pollitt, J. A. Carter, and International Child Development Steering Group. 2007. "Child Development: Risk Factors for Adverse Outcomes in Developing Countries." Lancet (London, England), vol. 369, no. 9556, pp. 145-157.

Walker, S. P., T. D. Wachs, S. Grantham-McGregor, M. M. Black, C. A. Nelson, S. L. Huffman, H. Baker-Henningham, S. M. Chang, J. D. Hamadani, B. Lozoff, J. M. Gardner, C. A. Powell, A. Rahman and L. Richter. 2011. "Inequality in Early Childhood: Risk and Protective Factors for Early Child Development." Lancet (London, England), vol. 378, no. 9799, pp. 1325-1338.

Whalley, L. J., I. J. Deary, C. L. Appleton, J. M. Starr. 2004. "Cognitive Reserve and the Neurobiology of Cognitive Aging." Ageing Research Reviews, vol. 3, no. 4, pp. 369382.

Wong, Y. N. 2012. "World Development Report. 2012: Gender Equality and Development." Forum for Development StudiesTaylor and Francis, pp. 435.

World Bank. 2015. World Bank Support to Early Childhood Development: An Independent Evaluation. World Bank Group, Washington, D.C. . 2013. The World Bank Annual Report 2013, World Bank, Washington, DC. . 2014. World Development Report 2015: Mind, Society, and Behavior. The World Bank, Washington, DC.

Ybarra, O., E. Burnstein, P. Winkielman, M. C. Keller, M. Manis, E. Chan, and J. Rodriguez. 2008. "Mental Exercising through Simple Socializing: Social Interaction 
Promotes General Cognitive Functioning." Personality and Social Psychology Bulletin, vol. 34, no. 2, pp. 248-259. 


"Human development" and "development" are inseparable, and now new evidence emerges that "brain development" is the key driving mechanism behind this association. The foundations of brain architecture are established early in life. Critical aspects of its structure begin to be shaped by experience before and soon after birth. The current report discusses brain development from a life-course perspective with a particular emphasis on early childhood development (ECD), skills formation, resilience and aging. There is mounting evidence that early and sustained investments in brain development have economic and social returns that can benefit current and future generations. This paper synthesizes knowledge across multiple disciplines and is weighted towards findings from brain sciences to encourage a new perspective on "human development" initiatives among policy makers and international development practitioners. The report discusses the role of social policies in shaping brain function and structure. The policy-relevant findings from brain sciences research can greatly enhance the ability to carry out cost-effective policies that foster human development over the life-course, eliminate extreme poverty and improve shared prosperity.

\section{ABOUT THIS SERIES:}

This series is produced by the Health, Nutrition, and Population Global Practice of the World Bank. The papers in this series aim to provide a vehicle for publishing preliminary results on HNP topics to encourage discussion and debate. The findings, interpretations, and conclusions expressed in this paper are entirely those of the author(s) and should not be attributed in any manner to the World Bank, to its affiliated organizations or to members of its Board of Executive Directors or the countries they represent. Citation and the use of material presented in this series should take into account this provisional character. For free copies of papers in this series please contact the individual author/s whose name appears on the paper. Enquiries about the series and submissions should be made directly to the Editor Martin Lutalo (mlutalo@ worldbank.org) or HNP Advisory Service (healthpop@worldbank.org, tel 202 473-2256).

For more information, see also www.worldbank.org/hnppublications.

1818 H Street, NW

Washington, DC USA 20433

Telephone: 2024731000

Facsimile: 2024776391

Internet: www.worldbank.org

E-mail: feedback@worldbank.org 